\title{
STRUCTURES OF GEOMETRIC QUOTIENT ORBIFOLDS OF THREE-DIMENSIONAL $G$-MANIFOLDS OF GENUS TWO
}

\author{
JUNGSOO KIM
}

\begin{abstract}
In this article, we will characterize structures of geometric quotient orbifolds of $G$-manifold of genus two where $G$ is a finite group of orientation preserving diffeomorphisms using the idea of handlebody orbifolds. By using the characterization, we will deduce the candidates of possible non-hyperbolic geometric quotient orbifolds case by case using W. Dunbar's work. In addition, if the $G$-manifold is compact, closed and the quotient orbifold's geometry is hyperbolic then we can show that the fundamental group of the quotient orbifold cannot be in the class $\mathcal{D}$.
\end{abstract}

\section{Introduction}

Let $M$ be a closed 3-manifold with a Heegaard splitting of genus two and $G$ be a finite group of orientation preserving diffeomorphisms acting on $M$, which preserves each handlebody of the Heegaard splitting. How can we determine the possible quotient orbifolds?

Since the $G$-action preserves each handlebody of the given Heegaard splitting, if we can describe the shape of the quotient of each handlebody by the $G$-action, then the quotient orbifold can be considered as a sum of two quotients of the handlebodies of the given Heegaard splitting. In [5], McCullough, Miller, and Zimmermann developed a deep theory which describes how finite groups act on handlebodies and how the quotient orbifolds, i.e., handlebody orbifolds, can be constructed.

In this article, we will characterize structures of quotient orbifolds of $G$ manifolds of genus two using the idea of handlebody orbifolds. By using the characterization, we will deduce the candidates of possible non-hyperbolic geometric quotient orbifolds case by case using W. Dunbar's work. In addition, if the $G$-manifold is compact, closed and the quotient orbifold's geometry is hyperbolic then we can show that the fundamental group of the quotient orbifold cannot be in the class $\mathcal{D}$, defined in Section 4 .

Received November 8, 2007; Revised January 28, 2008.

2000 Mathematics Subject Classification. Primary 57M99; Secondary 57S17.

Key words and phrases. orbifold, finite group action, handlebody orbifold, Heegaard splitting.

(C)2009 The Korean Mathematical Society 
In the theory of handlebody orbifolds, a graph of groups which satisfies certain conditions corresponds to a handlebody orbifold. At first, we will deduce all the possible $G$-admissible graphs of groups case by case as in the following lemma.

Lemma 2.9. Let $G$ be a finite group of orientation-preserving diffeomorphisms of the genus two handlebody. Then all the possible $G$-admissible graphs $(\Gamma, \mathcal{G})$ in standard form are as follows.

(1) For $G \cong \mathbb{Z}_{2}, \Gamma\left(\mathbb{Z}_{2}, 1\right), \Gamma\left(\mathbb{Z}_{2}, 1,\left(\mathbb{Z}_{2}, 2\right)\right)$ and $\Gamma\left(\mathbb{Z}_{2}, 1, \mathbb{Z}_{2}, 1, \mathbb{Z}_{2}\right)$.

(2) For $G \cong \mathbb{Z}_{3}, \Gamma\left(\mathbb{Z}_{3}, 1, \mathbb{Z}_{3}\right)$.

(3) For $G \cong \mathbb{Z}_{4}, \Gamma\left(\mathbb{Z}_{4}, 1, \mathbb{Z}_{2}\right)$.

(4) For $G \cong \mathbb{Z}_{6}, \Gamma\left(\mathbb{Z}_{3}, 1, \mathbb{Z}_{2}\right)$.

(5) For $G \cong \mathbb{D}_{2}, \Gamma\left(\mathbb{Z}_{2}, 1, \mathbb{D}_{2}\right), \Gamma\left(\mathbb{D}_{2}, 2\right)$ and $\Gamma\left(\mathbb{D}_{2}, 2, \mathbb{D}_{2}, 2, \mathbb{D}_{2}\right)$.

(6) For $G \cong \mathbb{D}_{3}, \Gamma\left(\mathbb{Z}_{2}, 1, \mathbb{Z}_{3}\right)$ and $\Gamma\left(\mathbb{D}_{3}, 2, \mathbb{D}_{3}\right)$.

(7) For $G \cong \mathbb{D}_{4}, \Gamma\left(\mathbb{D}_{2}, 2, \mathbb{D}_{4}\right)$.

(8) For $G \cong \mathbb{D}_{6}, \Gamma\left(\mathbb{D}_{2}, 2, \mathbb{D}_{3}\right)$.

In [5], there is a description of a method to construct a handlebody orbifold from a graph of groups which satisfies certain conditions. Using this, we obtain the following corollary.

Corollary 2.10. The handlebody orbifold quotients of G-actions on the genus two handlebody are exactly those given in Figure 2.

Using Lemma 2.9 and Corollary 2.10, we may deduce the following theorem which describes how the quotient orbifold can be constructed.

Theorem 3.3 (Main Theorem). Suppose that $M$ is a closed G-manifold of genus 2 with Heegaard decomposition $\left(M: V_{1}, V_{2}\right)$. Then the quotient orbifold is the sum of two handlebody orbifolds, where the two corresponding $G$-admissible graphs of handlebody orbifolds are the same type except in the following cases.

(1) $\mathcal{O}$ is a union of two handlebody orbifolds where one handlebody orbifold is modeled on $\Gamma\left(\mathbb{Z}_{2}, 1\right)$ and the other handlebody orbifold is modeled on $\Gamma\left(\mathbb{Z}_{2}, 1,\left(\mathbb{Z}_{2}, 2\right)\right)$.

(2) $\mathcal{O}$ is a union of two handlebody orbifolds where one handlebody orbifold is modeled on $\Gamma\left(\mathbb{Z}_{2}, 1, \mathbb{D}_{2}\right)$ and the other handlebody orbifold is modeled on $\Gamma\left(\mathbb{D}_{2}, 2, \mathbb{D}_{2}, 2, \mathbb{D}_{2}\right)$.

(3) $\mathcal{O}$ is a union of two handlebody orbifolds where one handlebody orbifold is modeled on $\Gamma\left(\mathbb{Z}_{2}, 1, \mathbb{Z}_{3}\right)$ and the other handlebody orbifold is modeled on $\Gamma\left(\mathbb{D}_{3}, 2, \mathbb{D}_{3}\right)$.

In Lemma 4.2, we will see that the underlying spaces of the quotient orbifolds are $S^{3}$, except in a few cases. In [2], Dunbar classified all the non-hyperbolic geometric closed orbifolds whose underlying spaces are $\mathbb{S}^{3}$, and gave explicit descriptions of figures. Theorem 3.3 enables us to determine the singular locus of each quotient orbifold. In Section 4, we use this information together with Dunbar's list of orbifolds to work out the possible candidates for nonhyperbolic geometric quotients. 

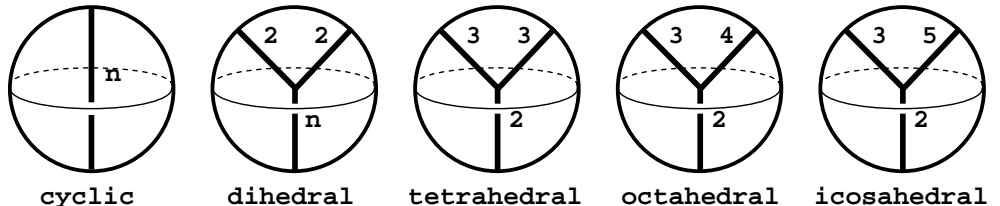

Figure 1. The quotients of the 3 -ball by spherical groups

In [3], Klimenko and Kopteva classified all the Kleinian orbifolds whose orbifold fundamental groups are contained in the class $\mathcal{D}$. In particular, they listed the explicit descriptions of figures of those orbifolds and determined the exact conditions for the cases of compact orbifolds. By using Theorem 3.3, we may restrict the singular locus of each quotient orbifold. So by checking all the possible cases of Theorem 3.3 using the figures of Klimenko and Kopteva and the conditions for the cases of compact orbifolds, we may deduce that no fundamental group of a quotient orbifold is contained in the class $\mathcal{D}$ (see Theorems 5.5-5.9).

Acknowledgement. I would like to thank to Darryl McCullough for pointing out one missing case of Lemma 2.9 and for thoughtful advice on polishing my paper.

\section{Handlebody orbifolds}

Let $G$ be a finite group of orientation preserving diffeomorphisms acting on a handlebody $V$. Let $D$ be a 2-dimensional properly embedded disk in $V$ such that $\partial D=D \cap \partial V$ is a nontrivial closed curve on $\partial V$. By the equivariant loop theorem and equivariant Dehn's lemma (see [6]), we may assume that $x(D)=D$ or $x(D) \cap D=\emptyset$ for all $x \in G$. When cutting $V$ along the system of disjoint disks $G(D)$, that is, removing the interior of a $G$-invariant regular neighborhood of $G(D)$ (which is a collection of 1-handles: products of a 2disk with an interval), we get again a collection of handlebodies of lower genus where $G$ acts. Applying inductively the above procedure of cutting along disks, we finally end up with a collection of disjoint 3-balls where $G$ acts. Thus the quotient orbifold $\mathcal{H}:=V / G$ is built up from orbifolds that are quotients of 3balls by finite group of homeomorphisms (their stabilizers in G), connected by finite cyclic quotients of 1-handles (1-handle orbifolds) which are the projection of the removed regular neighborhoods of the disks (the first type of orbifold in Figure 1).

The finite groups that can act preserving orientation on the 3-ball or the 2-sphere are the finite subgroups of the orthogonal group $S O(3)$ : cyclic $\mathbb{Z}_{n}$, dihedral $\mathbb{D}_{n}$, tetrahedral $\mathbb{A}_{4}$, octahedral $\mathbb{S}_{4}$ and dodecahedral $\mathbb{A}_{5}$, which we will call the spherical groups. The possible quotient orbifolds from 3-balls are listed in Figure 1; 
Definition 2.1 ([9]). A handlebody orbifold consists of finitely many orbifolds as in Figure 1 (i.e., quotients of finite orthogonal group actions on the 3-ball) connected by 1-handle orbifolds respecting the singular axes and their orders, and such that topologically the outcome is an orientable handlebody.

By Definition 2.1, the singular locus in the handlebody orbifold is a trivalent graph.

In general, we can say that the quotient orbifold $V / G$ is a handlebody orbifold by the following proposition.

Proposition 2.2 ([9, Proposition 1]). The quotients of handlebodies by finite group actions are the handlebody orbifolds.

The concept of $G$-admissible graph of groups is the key idea to classify the finite group actions on handlebodies. To define it, we need to introduce the concept of graph of groups. See details in [8].

Consider a finite graph of finite groups $(\Gamma, \mathcal{G})$.

Definition 2.3 ([7]). If $G$ is a finite group then a homomorphism $\rho: \pi_{1}(\Gamma, \mathcal{G}) \rightarrow$ $G$ is said to be finite-injective provided that $\rho$ is one-to-one on each finite subgroup of $\pi_{1}(\Gamma, \mathcal{G})$; equivalently, $\rho$ is injective on each vertex group of $\pi_{1}(\Gamma, \mathcal{G})$.

In [5] a set of normalized conditions for a graph of groups is given which is used to study group actions on handlebodies.

Definition $2.4([7])$. A graph of groups $(\Gamma, \mathcal{G})$ is called $G$-admissible if it satisfies the normalized conditions and there is a finite-injective epimorphism from $\pi_{1}(\Gamma, \mathcal{G})$ to $G$.

Definition $2.5([7])$. The Euler characteristic of a graph of groups $(\Gamma, \mathcal{G})$ is defined as follows.

$$
\chi(\Gamma, \mathcal{G})=\sum \frac{1}{\left|G_{v}\right|}-\sum \frac{1}{\left|G_{e}\right|},
$$

where $G_{v}$ is the vertex group of vertex $v$, and $G_{e}$ is the edge group of edge $e$ in $(\Gamma, \mathcal{G})$.

Proposition 2.6 ([5, Theorem 6.1]). Let $G$ be a finite group. There is an orientation-preserving effective G-action on the handlebody $V_{g}$ of genus $g$ if and only if there is a $G$-admissible graph of groups $(\Gamma, \mathcal{G})$ such that

$$
g=1-|G| \chi(\Gamma, \mathcal{G}) .
$$

By Propositions 2.2 and 2.6, we can say that a $G$-admissible graph of groups $(\Gamma, \mathcal{G})$ whose Euler characteristic satisfies the equation $g=1-|G| \chi(\Gamma, \mathcal{G})$ realizes a handlebody orbifold.

Proposition 2.7 ([5, Theorem 7.2]). Let $G$ be a finite group of orientationpreserving diffeomorphisms of the orientable 3 -dimensional handlebody of genus $g \geq 2$. Then the order of $G$ is less than or equal to $12(g-1)$. 
By Proposition 2.6, if $g=2$, then

$$
\chi(\Gamma, \mathcal{G})=-1 /|G|,
$$

and by Proposition 2.7 , if $|G| \geq 2$, then

$$
-\frac{1}{2} \leq \chi(\Gamma, \mathcal{G}) \leq-\frac{1}{12}
$$

Proposition 2.8 ([5, Theorem 8.2(b)]). The finite groups which act on $V_{2}$ are the subgroups of $\mathbb{D}_{4}$ and $\mathbb{D}_{6}$ up to isomorphism.

By Proposition 2.8, the possible finite groups which act on $V_{2}$ are cyclic groups of order 2, 3, 4, 6 and dihedral groups of orders 4, 6, 8, 12. Therefore the possible Euler characteristics of $G$-admissible graphs of groups $(\Gamma, \mathcal{G})$ are $-\frac{1}{2},-\frac{1}{3},-\frac{1}{4},-\frac{1}{6},-\frac{1}{8}$ and $-\frac{1}{12}$.

The construction of the handlebody orbifold $V(\Gamma, \mathcal{G})$ from a graph of groups $(\Gamma, \mathcal{G})$ is described on pp. $389-390$ of [5]. From section 3 of [5], we have

$$
\pi_{1}^{\text {orb }}(V(\Gamma, \mathcal{G})) \cong \pi_{1}(\Gamma, \mathcal{G})
$$

We will use the notations $\Gamma(A, n, B), \Gamma(A, m, B, n, C), \Gamma(A, n)$ and $\Gamma(A, 1$, $(B, n))$ to denote the graphs of groups whose fundamental groups are isomorphic to $A * \mathbb{Z}_{n} B, A * \mathbb{Z}_{m} B * \mathbb{Z}_{n} C, A * \mathbb{Z}_{n}$ and $A *\left(B * \mathbb{Z}_{n}\right)$.

Lemma 2.9. Let $G$ be a finite group of orientation-preserving diffeomorphisms of the genus two handlebody. Then all the possible $G$-admissible graphs $(\Gamma, \mathcal{G})$ in standard form are as follows:

(1) For $G \cong \mathbb{Z}_{2}, \Gamma\left(\mathbb{Z}_{2}, 1\right), \Gamma\left(\mathbb{Z}_{2}, 1,\left(\mathbb{Z}_{2}, 2\right)\right)$ and $\Gamma\left(\mathbb{Z}_{2}, 1, \mathbb{Z}_{2}, 1, \mathbb{Z}_{2}\right)$.

(2) For $G \cong \mathbb{Z}_{3}, \Gamma\left(\mathbb{Z}_{3}, 1, \mathbb{Z}_{3}\right)$.

(3) For $G \cong \mathbb{Z}_{4}, \Gamma\left(\mathbb{Z}_{4}, 1, \mathbb{Z}_{2}\right)$.

(4) For $G \cong \mathbb{Z}_{6}, \Gamma\left(\mathbb{Z}_{3}, 1, \mathbb{Z}_{2}\right)$.

(5) For $G \cong \mathbb{D}_{2}, \Gamma\left(\mathbb{Z}_{2}, 1, \mathbb{D}_{2}\right), \Gamma\left(\mathbb{D}_{2}, 2\right)$ and $\Gamma\left(\mathbb{D}_{2}, 2, \mathbb{D}_{2}, 2, \mathbb{D}_{2}\right)$.

(6) For $G \cong \mathbb{D}_{3}, \Gamma\left(\mathbb{Z}_{2}, 1, \mathbb{Z}_{3}\right)$ and $\Gamma\left(\mathbb{D}_{3}, 2, \mathbb{D}_{3}\right)$.

(7) For $G \cong \mathbb{D}_{4}, \Gamma\left(\mathbb{D}_{2}, 2, \mathbb{D}_{4}\right)$.

(8) For $G \cong \mathbb{D}_{6}, \Gamma\left(\mathbb{D}_{2}, 2, \mathbb{D}_{3}\right)$.

Proof. In cases (2), (3) and (4), use the table in the proof of Theorem 7.3(c) in $[5]$.

In cases (5), (6), (7) and (8) we can see that $|G| \geq 4$, so the possible Euler characteristics of $G$-admissible graphs $(\Gamma, \mathcal{G})$ are greater than or equal to $-\frac{1}{4}$.

We will use Chart B from p. 401 of [5], which lists all the graphs $(\Gamma, \mathcal{G})$ in standard form which satisfy the normalized conditions with $-\frac{1}{4} \leq \chi(\Gamma, \mathcal{G}) \leq$ $-\frac{1}{12}$.

In case (5), there are $12 G$-admissible graphs of Euler Characteristic $-\frac{1}{4}$ in Chart B in p. 401 of $[5]$. But $\Gamma\left(\mathbb{Z}_{2}, 1, \mathbb{Z}_{4}\right), \Gamma\left(\mathbb{D}_{3}, 2, \mathbb{D}_{6}\right), \Gamma\left(\mathbb{D}_{3}, 2, \mathbb{A}_{4}\right), \Gamma\left(\mathbb{D}_{4}, 2\right.$, $\left.\mathbb{D}_{4}\right), \Gamma\left(\mathbb{S}_{4}, 3, \mathbb{S}_{4}\right), \Gamma\left(\mathbb{A}_{4}, 3\right), \Gamma\left(\mathbb{D}_{3}, 3, \mathbb{A}_{4}, 3, \mathbb{D}_{3}\right), \Gamma\left(\mathbb{D}_{3}, 3, \mathbb{S}_{4}, 4, \mathbb{D}_{4}\right)$ and $\Gamma\left(\mathbb{D}_{3}, 3, \mathbb{S}_{5}\right.$, $\left.5, \mathbb{D}_{5}\right)$ are impossible since some vertex group of each graph has some element of order larger than 2 , so this element cannot be an injective image in $\mathbb{D}_{2}$. In 
particular, we do not consider $\Gamma\left(\mathbb{D}_{2}, 2, \mathbb{D}_{k}, k, \mathbb{D}_{k}\right)$ with $k>2$ since the edge group $\mathbb{Z}_{k}$ realizes a 1 -handle with its core of index $k>2$ in the corresponding handlebody orbifold, but this is impossible for a $\mathbb{D}_{2}$-action.

In case (6), there are $7 \mathrm{G}$-admissible graphs of Euler Characteristic $-\frac{1}{6}$ in Chart B in p. 401 of $[5]$. But $\Gamma\left(\mathbb{D}_{2}, 2, \mathbb{D}_{6}\right), \Gamma\left(\mathbb{D}_{2}, 2, \mathbb{A}_{4}\right), \Gamma\left(\mathbb{A}_{4}, 3, \mathbb{A}_{4}\right), \Gamma\left(\mathbb{S}_{4}, 4, \mathbb{S}_{4}\right)$ and $\Gamma\left(\mathbb{A}_{5}, 5, \mathbb{A}_{5}\right)$ are impossible since some vertex group of each graph has order larger than $6=|G|=\left|\mathbb{D}_{3}\right|$, so this vertex group cannot be an injective image in $G$. Case (7) is similar.

In case (8), there are $4 G$-admissible graphs of Euler Characteristic $-\frac{1}{12}$ in Chart B in p. 401 of $[5]$. But $\Gamma\left(\mathbb{D}_{4}, 4, \mathbb{S}_{4}\right)$ and $\Gamma\left(\mathbb{D}_{5}, 5, \mathbb{A}_{5}\right)$ are impossible since some vertex group of each graph has order larger than $12=|G|$ where $G \cong \mathbb{D}_{6}$, so this vertex group cannot be an injective image in $G . \Gamma\left(\mathbb{D}_{3}, 3, \mathbb{A}_{4}\right)$ is also impossible since $\left|\mathbb{A}_{4}\right|=|G|$ but $\mathbb{A}_{4}$ does not embed in $G \cong \mathbb{D}_{6}$.

Now we consider case (1).

Suppose that $(\Gamma, \mathcal{G})$ has only one vertex. If there is an edge with trivial edge group, then since $\chi(\Gamma, \mathcal{G})=-\frac{1}{2}$, it is the only edge, and we have $\Gamma\left(\mathbb{Z}_{2}, 1\right)$. If no edge has trivial edge group, then since $\chi(\Gamma, \mathcal{G})=-\frac{1}{2}$, there must be exactly two $\mathbb{Z}_{2}$-edges attached to the vertex, but then the graph cannot be the singular set of a 3-orbifold.

Suppose now that $(\Gamma, \mathcal{G})$ has two vertices. Necessarily they are connected by a trivial edge, and there must be one more $\mathbb{Z}_{2}$-edge to produce $\chi(\Gamma, \mathcal{G})=-\frac{1}{2}$. Since the graph is in standard form, both ends of the $\mathbb{Z}_{2}$-edge are attached to one of the vertices, giving the case of $\Gamma\left(\mathbb{Z}_{2}, 1,\left(\mathbb{Z}_{2}, 2\right)\right)$.

Finally, suppose that $(\Gamma, \mathcal{G})$ has $n \geq 3$ vertices. These must be connected by at least $n-1$ trivial edges, so $-\frac{1}{2}=\chi(\Gamma, \mathcal{G}) \leq \frac{n}{2}-(n-1)=1-\frac{n}{2}$. This implies that $n=3$ and $(\Gamma, \mathcal{G})=\Gamma\left(\mathbb{Z}_{2}, 1, \mathbb{Z}_{2}, 1, \mathbb{Z}_{2}\right)$.

Corollary 2.10. The handlebody orbifold quotients of G-actions on the genus two handlebody are exactly those given in Figure 2.

Note that the handlebody orbifolds of Figure 2 correspond to the G-admissible graphs.

\section{Finite group actions on $G$-manifolds of genus two}

Definition 3.1 ([9]). A closed 3-manifold $M$ is called a $G$-manifold of genus $g$ if it admits an action of the finite group $G$ and $g$ is the minimal genus of a Heegaard splitting of $M$ for which both handlebodies are invariant under the $G$-action. This minimal $g$ is called the equivariant Heegaard genus of the action.

Definition $3.2([9])$. A Heegaard decomposition of a closed orientable orbifold $\mathcal{O}$ is a decomposition of the orbifold into two handlebody orbifolds $\mathcal{H}_{1}$ and $\mathcal{H}_{2}$ intersecting in their common boundary (a 2-orbifold).

Suppose that $M$ is a $G$-manifold of genus 2 and the Heegaard decomposition of $M$ is $\left(M: V_{1}, V_{2}\right)$ with gluing map $f: \partial V_{1} \rightarrow \partial V_{2}$. From now on, the 


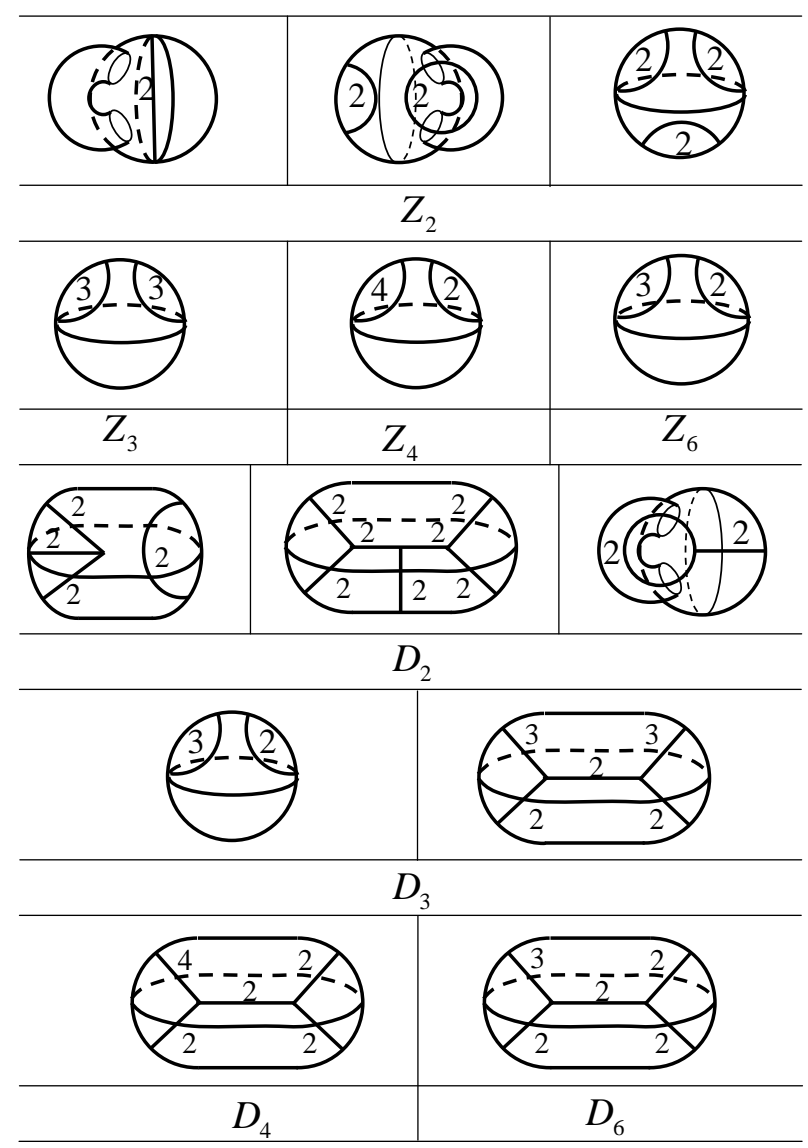

FiguRe 2. All handlebody orbifolds from genus two handlebody.

Heegaard genus is assumed to be 2 unless otherwise specified. Then the quotient orbifold $\mathcal{O}:=M / G$ is the union of two handlebody orbifolds $\mathcal{H}_{1}:=V_{1} / G$ and $\mathcal{H}_{2}:=V_{2} / G$ by the gluing map $\phi: \partial \mathcal{H}_{1} \rightarrow \partial \mathcal{H}_{2}$ induced from $f$. So using Corollary 2.10 we get the following theorem.

Theorem 3.3 (Main Theorem). Suppose that $M$ is a closed G-manifold of genus 2 with Heegaard decomposition $\left(M: V_{1}, V_{2}\right)$. Then the quotient orbifold is the sum of two handlebody orbifolds, where the two corresponding $G$-admissible graphs of handlebody orbifolds are the same type except in the following cases.

(1) $\mathcal{O}$ is a union of two handlebody orbifolds where one handlebody orbifold is modeled on $\Gamma\left(\mathbb{Z}_{2}, 1\right)$ and the other handlebody orbifold is modeled on $\Gamma\left(\mathbb{Z}_{2}, 1,\left(\mathbb{Z}_{2}, 2\right)\right)$. 
(2) $\mathcal{O}$ is a union of two handlebody orbifolds where one handlebody orbifold is modeled on $\Gamma\left(\mathbb{Z}_{2}, 1, \mathbb{D}_{2}\right)$ and the other handlebody orbifold is modeled on $\Gamma\left(\mathbb{D}_{2}, 2, \mathbb{D}_{2}, 2, \mathbb{D}_{2}\right)$.

(3) $\mathcal{O}$ is a union of two handlebody orbifolds where one handlebody orbifold is modeled on $\Gamma\left(\mathbb{Z}_{2}, 1, \mathbb{Z}_{3}\right)$ and the other handlebody orbifold is modeled on $\Gamma\left(\mathbb{D}_{3}, 2, \mathbb{D}_{3}\right)$.

Proof. Consider the gluing map $\phi: \partial \mathcal{H}_{1} \rightarrow \partial \mathcal{H}_{2}$. Since $G$ acts on $V_{1}$ and $V_{2}$ simultaneously and preserves $V_{1}$ and $V_{2}, \mathcal{H}_{1}$ and $\mathcal{H}_{2}$ are both handlebody orbifolds of G-actions. Since $\phi\left(\partial \mathcal{H}_{1}\right)=\partial \mathcal{H}_{2}$, the numbers of singular points in $\partial \mathcal{H}_{1}$ and $\partial \mathcal{H}_{2}$ are the same and $\phi$ sends singular points to singular points of the same index. Using the classification given in Corollary 2.10, we observe that $\mathcal{H}_{1}$ and $\mathcal{H}_{2}$ are the same orbifold homeomorphism type except in some cases of $G \cong \mathbb{Z}_{2}, \mathbb{D}_{2}$ and $\mathbb{D}_{3}$. In the case of $\mathbb{Z}_{2}$, some unions of $\mathcal{H}_{1}$ and $\mathcal{H}_{2}$ obtained from $\Gamma\left(\mathbb{Z}_{2}, 1\right)$ and $\Gamma\left(\mathbb{Z}_{2}, 1,\left(\mathbb{Z}_{2}, 2\right)\right)$ are also possible. In the case of $\mathbb{D}_{2}$, some unions of $\mathcal{H}_{1}$ and $\mathcal{H}_{2}$ obtained from $\Gamma\left(\mathbb{Z}_{2}, 1, \mathbb{D}_{2}\right)$ and $\Gamma\left(\mathbb{D}_{2}, 2, \mathbb{D}_{2}, 2, \mathbb{D}_{2}\right)$ are also possible. In the case of $\mathbb{D}_{3}$, some unions of $\mathcal{H}_{1}$ and $\mathcal{H}_{2}$ obtained from $\Gamma\left(\mathbb{Z}_{2}, 1, \mathbb{Z}_{3}\right)$ and $\Gamma\left(\mathbb{D}_{3}, 2, \mathbb{D}_{3}\right)$ are also possible.

\section{Non-hyperbolic geometric quotients of $G$-manifolds of genus two}

In [2], W. Dunbar classified non-hyperbolic geometric 3-orbifolds using the following classification theorem.

Proposition 4.1 ([2, Theorem 1]). Suppose that $\mathcal{O}^{3}$ is a geometric orbifold. Then exactly one of the following holds:

(1) $\mathcal{O}^{3}$ is a hyperbolic orbifold.

(2) $\mathcal{O}^{3}$ is a Seifert fibered orbifold with base $\mathcal{O}^{2}$, in which case one of the following descriptions applies (both may apply if $\mathcal{O}^{3}$ fibers in more than one way) :

(a) if $\partial X^{2}=\emptyset\left(X^{2}\right.$ denotes the underlying space of $\left.\mathcal{O}^{2}\right)$, then $X^{3}$ is a closed Seifert fibered manifold, and $\Sigma_{\mathcal{O}^{3}}$ consists of fibers (labeled with various integers $>1)$;

(b) if $\partial X^{2}$ has $n$ components $(n>0)$, then $X^{3}$ is the result of gluing solid tori to a Seifert fibered manifold with $n$ boundary tori, such that the meridians of the tori are glued to fibers on the boundary. $\Sigma_{\mathcal{O}^{3}}$ consists of fibers, plus a Montesinos link (labeled $\ll 2 \gg$, and slightly modified) inside each added solid torus.

(3) $\mathcal{O}^{3}$ is a solvorbifold; in particular, it fibers over either $\mathbb{S}^{1}$ or $\mathbb{S}^{1} /$ reflection, with fiber either $T^{2}$ or $T^{2} /\left(180^{\circ}\right.$ rotation fixing 4 points $)=\left(\mathbb{S}^{1} \times \mathbb{S}^{1}\right) /$ (reflection of both factors).

(4) $\mathcal{O}^{3}$ is one of the 12 Euclidean orbifolds (11 with $X^{3} \simeq \mathbb{S}^{3}$ and 1 with $X^{3} \simeq \mathbb{R P}^{3}$ ) or 18 spherical orbifolds (all with $X^{3} \simeq \mathbb{S}^{3}$ ) which are not fibered over 2 -orbifolds. 
Dunbar worked out a complete classification of non-hyperbolic geometric 3orbifolds with underlying space $\mathbb{S}^{3}$. They are listed in his explicit descriptions of figures on pp. 81-86, pp. 89-93, p. 97 of [2]. Thus all closed geometric orbifolds with underlying space $\mathbb{S}^{3}$ not in Dunbar's figures are hyperbolic (see the details in the last paragraph of p. 69 in [2]).

Lemma 4.2. Suppose that $M$ is a closed $G$-manifold of genus two where $G$ is a group of finite-order orientation preserving diffeomorphisms. Then the underlying space of the quotient orbifold $\mathcal{O}:=M / G$ is either

(1) $\mathbb{S}^{3}$, or

(2) $L(p, q)$ in which case any one of both handlebody orbifolds is obtained from $\Gamma\left(\mathbb{Z}_{2}, 1\right), \Gamma\left(\mathbb{Z}_{2}, 1,\left(\mathbb{Z}_{2}, 2\right)\right)$ or $\Gamma\left(\mathbb{D}_{2}, 2\right)$.

Proof. Consider the cases any one of both handlebody orbifolds is obtained from $\Gamma\left(\mathbb{Z}_{2}, 1\right), \Gamma\left(\mathbb{Z}_{2}, 1,\left(\mathbb{Z}_{2}, 2\right)\right)$ or $\Gamma\left(\mathbb{D}_{2}, 2\right)$. Then the underlying spaces of both handlebody orbifolds are homeomorphic to solid tori by Theorem 3.3 and Corollary 2.10 , so $\mathcal{O}$ is homeomorphic to $L(p, q)$ for some $p, q\left(\mathbb{S}^{3}\right.$ and $\mathbb{S}^{2} \times \mathbb{S}^{1}$ are included). Otherwise, the underlying spaces of both handlebody orbifolds are homeomorphic to $\mathbb{B}^{3}$, so the underlying space of $\mathcal{O}$ is a union of two 3-balls by identifying their boundaries, i.e., $\mathbb{S}^{3}$.

In the remainder of this section, we will determine the quotient orbifolds that are non-hyperbolic and geometric with underlying space $\mathbb{S}^{3}$.

From now on, let $M$ be a $G$-manifold of genus two, where $G$ is finite and orientation-preserving. Fix an invariant Heegaard decomposition $\left(M: V_{1}, V_{2}\right)$. We will denote the quotient orbifolds $V_{1} / G, V_{2} / G$, and $M / G$ by $\mathcal{H}_{1}, \mathcal{H}_{2}$, and $\mathcal{O}$ respectively.

Suppose that both of $\mathcal{H}_{1}$ and $\mathcal{H}_{2}$ are rational tangle orbifolds. Since $\mathcal{O}$ is obtained by identifying the boundaries of $\mathcal{H}_{1}$ or $\mathcal{H}_{2}$, it has underlying space $\mathbb{S}^{3}$ and singular set a knot or link of at most two bridges. We will refer to such a knot of link as a " $(\leq 2)$-bridge knot or link." We remind the reader that for a 2-bridge link, each component is a 1-bridge knot so is a trivial knot.

We call a orbifold a rational tangle orbifold if it its underlying space is a 3-ball and the singular loci form a rational tangle. We say that two rational tangle orbifolds are the same if the indices of the singular loci of one orbifold are the same as those of the other. From Corollary 2.10 and Theorem 3.3, we have the following result immediately:

Lemma 4.3. Suppose that one of $\mathcal{H}_{1}$ or $\mathcal{H}_{2}$ is a rational tangle orbifold, then the other is the same rational tangle orbifold, so the singular locus of $\mathcal{O}$ is a $(\leq 2)$-bridge knot or link. In particular, if the indices of the two components of the singular locus of $\mathcal{H}_{1}$ are different, then the singular locus is a 2-bridge link, and each component is unknotted.

In many of Dunbar's figures, the singular loci form a "Montesinos graph." The definition of a Montesinos graph is similar to that of a Montesinos link, 


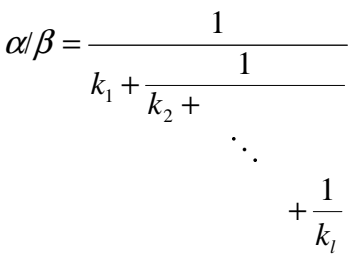

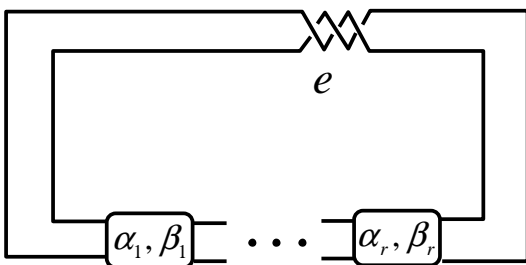

FiguRE 3. The tangled graph with parameter $(55,235)$

but the Montesinos graph uses "tangled graphs" which are a bit different from rational tangles (see Figure 3). In Dunbar's article the parameter $(m, n)$ of each rational tangle means the continued faction of $m / n$, but in many other authors' articles the parameter $(m, n)$ of each rational tangle means the continued fraction of $n / m$. We will use Dunbar's notation to represent rational tangles for convenience. If a tangled graph has coprime parameter $(m, n)$, then it is exactly same as a rational tangle with same parameter. If $\operatorname{gcd}(m, n) \neq 1$, then there is a strut of index $\operatorname{gcd}(m, n)$ which connects the strands in the innermost twist as in Figure 4. For the parameter $(m, n)$ we will require that $m, n \in \mathbb{Z},|m| \leq n / 2$ and $n>1$. The tangled graph with parameter $(-m, n)$ is obtained by reversing all crossings in the tangled graph with parameter $(m, n)$. All the indices of singular loci of Montesinos graph are 2 except the struts from tangled graphs with parameters $(m, n)$, where $\operatorname{gcd}(m, n)>2$. In Montesinos graphs, a tangled graph with parameter $(0, n)$ is also possible, which is a tangle with two horizontal strands with a vertical strut of index $n$ which connect them (see Figure 5). See section 4 of [2] for more details.

In some cases when the Montesinos graphs are Montesinos links, we need to determine the bridge number of the singular locus. In Dunbar's figures, there are at most three tangled graphs with parameters in Montesinos graphs. In the cases of Montesinos links with $r=3$ rational tangles (so the parameters $\left(m_{i}, n_{i}\right)$ are coprime for all $\left.i\right)$, we may say that the bridge number is 3 by Lemma 4.4 .

Lemma 4.4 ([1, Theorem 1.1]). If $L=m\left(0 \mid e ;\left(\alpha_{1}, \beta_{1}\right), \ldots,\left(\alpha_{r}, \beta_{r}\right)\right)$ is a Montesinos link with $r \geq 3$ rational tangles, then $w(L)=b(L)=r$, where $w(L)$ is 


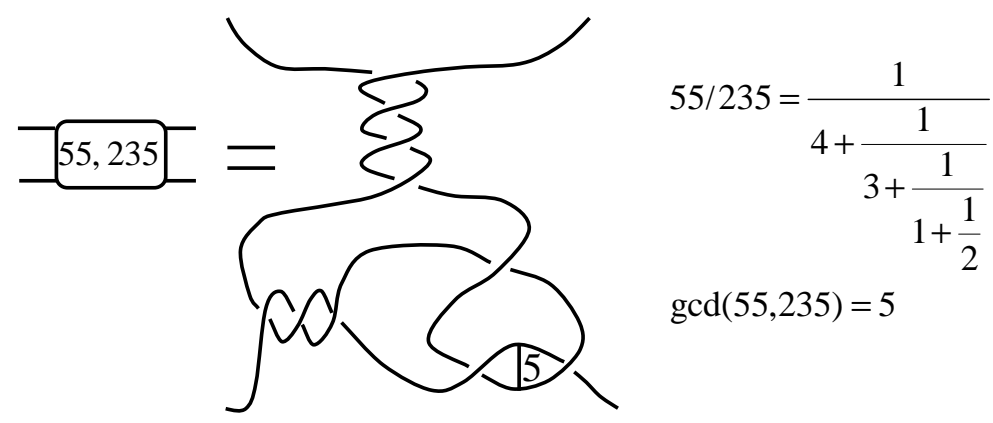

Figure 4. The tangled graph

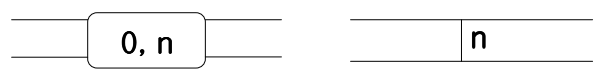

Figure 5 . The tangled graph with parameter $(0, n)$

the minimal number of meridian generators necessary to generate $\pi_{1}\left(\mathbb{S}^{3}-L\right)$ and $b(L)$ is the bridge number of $L$.

From Theorem 4.5 to Theorem 4.16 we will consider the cases when the underlying spaces of $\mathcal{O}$ are homeomorphic to $\mathbb{S}^{3}$ (see Lemma 4.2). So we will find appropriate candidates of $\mathcal{O}$ in Dunbar's figures case by case using Theorem 3.3. In each theorem, we will describe some possible orbifolds for each case. But the converse may not be true. That is, some orbifold $\mathcal{O}^{\prime}$ can be in the list of candidates, but there might not exist a finite group action $G$ and a $G$-manifold $M$ of genus two such that $\mathcal{O}^{\prime} \cong M / G$.

In each figure, the page number shown is the page number where the figure appears in [2] and the type of the orbifold following the page number is the type of orbifold in Proposition 4.1. Each figure is just a redrawing of Dunbar's figure, but not all the information in the original figure appears. For more information about the orbifold in each figure (its base orbifold, its geometry, whether it has an orientation reversing self-diffeomorphism, etc) see the original figure of Dunbar. If the orbifold has a bona fide mirror image (another oriented orbifold such that there is an orientation-reversing diffeomorphism between the two, but no orientation-preserving diffeomorphism), only one of the pair is pictured (see section 6 of [2]). In the cases when coefficients must be prescribed, the prescribed coefficients appear with an emboldened font in a rectangular box.

Theorem 4.5. Suppose that $\mathcal{O}$ is non-hyperbolic and geometric. If both $\mathcal{H}_{1}$ and $\mathcal{H}_{2}$ are handlebody orbifolds modeled on $\Gamma\left(\mathbb{Z}_{2}, 1, \mathbb{Z}_{3}\right)$ (so $G \cong \mathbb{Z}_{6}$ or $\mathbb{D}_{3}$ ), then $\mathcal{O}$ is one of the orbifolds in Figure 6.

Proof. By Lemma 4.3, the singular locus of $\mathcal{O}$ is a two bridge link with one component having index 2 and the other having index 3 . 


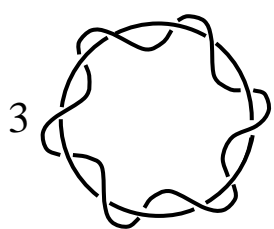

p.81 (type 2a)

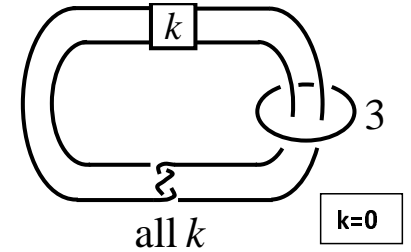

p.84 (type 2b)

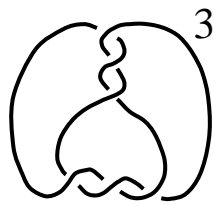

p.86 (type 4)

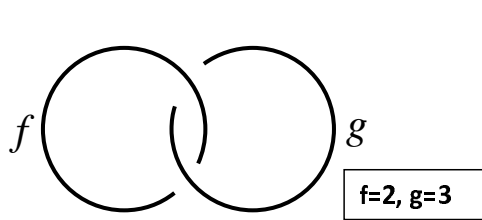

p.89 (type 2a)

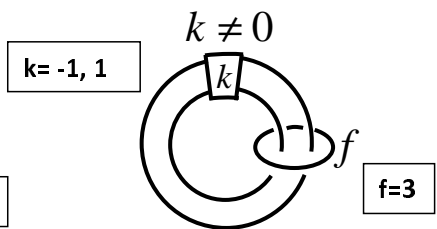

p.89 (type 2a)

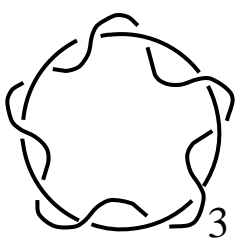

p.90 (type 2a)

p.90 (type 2a)

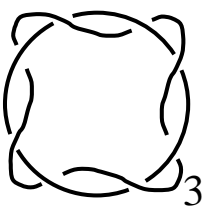

p.90 (type 2a)
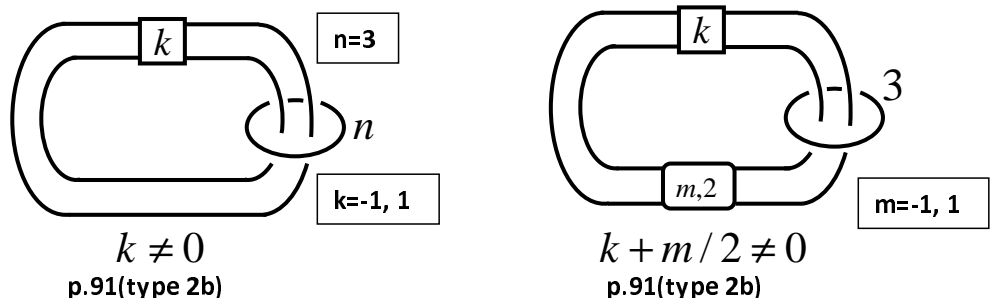

Figure $6 . \mathcal{H}_{1}$ and $\mathcal{H}_{2}: \Gamma\left(\mathbb{Z}_{3}, 1, \mathbb{Z}_{2}\right)$. In the last figure, the coefficients $k$ and $m$ need to be determined so that the component is an unknot.

Theorem 4.6. Suppose $\mathcal{O}$ is geometric. If $\mathcal{H}_{1}$ and $\mathcal{H}_{2}$ are handlebody orbifolds modeled on $\Gamma\left(\mathbb{Z}_{2}, 1, \mathbb{Z}_{3}\right)$ and $\Gamma\left(\mathbb{D}_{2}, 2, \mathbb{D}_{3}\right)$ respectively $\left(\right.$ so $\left.G \cong \mathbb{D}_{3}\right)$, then $\mathcal{O}$ is hyperbolic.

Proof. In this case, the singular locus is connected and consists of two vertices of degree 3 and three edges, one is of index 3 and the others are of index 2 . In particular, all the edges are non-separating edges in the graph of the singular locus. Since such orbifolds do not exist in Dunbar's list of figures, the orbifold must be hyperbolic. 


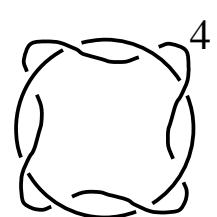

p.81 (type 2a)

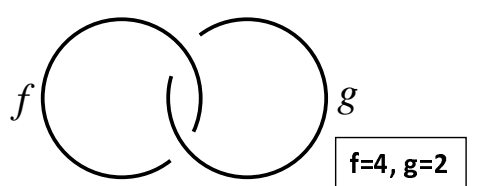

p.89 (type 2a)

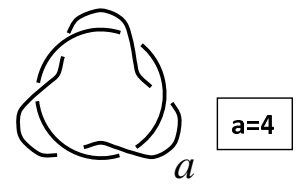

p.90 (type 2a)

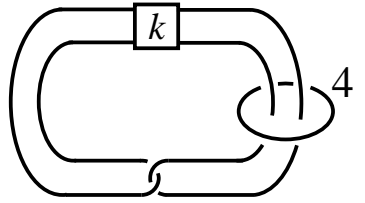

all $k$

p.83 (type 2b)

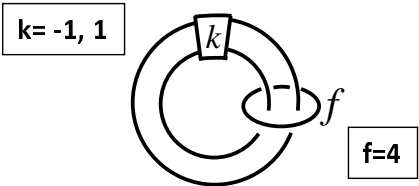

p.89 (type 2a)

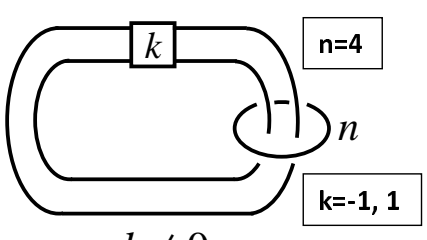

$k \neq 0$

p.91(type 2b)

FiguRE $7 . \mathcal{H}_{1}$ and $\mathcal{H}_{2}: \Gamma\left(\mathbb{Z}_{4}, 1, \mathbb{Z}_{2}\right)$. In the second figure, the coefficient $k$ need to be determined so that the component is an unknot.

Theorem 4.7. Suppose that $\mathcal{O}$ is non-hyperbolic and geometric. If both $\mathcal{H}_{1}$ and $\mathcal{H}_{2}$ are handlebody orbifolds modeled on $\Gamma\left(\mathbb{Z}_{4}, 1, \mathbb{Z}_{2}\right)$ (so $\left.G \cong \mathbb{Z}_{4}\right)$, then $\mathcal{O}$ is one of the orbifolds in Figure $\%$.

Proof. A similar argument shows the result.

Theorem 4.8. Suppose that $\mathcal{O}$ is non-hyperbolic and geometric. If both $\mathcal{H}_{1}$ and $\mathcal{H}_{2}$ are handlebody orbifolds modeled on $\Gamma\left(\mathbb{Z}_{3}, 1, \mathbb{Z}_{3}\right)$ (so $G \cong \mathbb{Z}_{3}$ ), then $\mathcal{O}$ is one of the orbifolds in Figure 8.

Proof. By Lemma 4.3, the singular locus is a $(\leq 2)$-bridge knot or link. If the singular locus is a knot, then the index of the singular locus is 3 and if the singular locus is a link, then the indices of the components are both 3 . In the case of the torus knot, we may use the fact that the bridge number of a $(p, q)$ torus knot is the minimum of $p$ and $q$.

Theorem 4.9. Suppose that $\mathcal{O}$ is non-hyperbolic and geometric. If both $\mathcal{H}_{1}$ and $\mathcal{H}_{2}$ are handlebody orbifolds modeled on $\Gamma\left(\mathbb{Z}_{2}, 1, \mathbb{Z}_{2}, 1, \mathbb{Z}_{2}\right)$ (so $G \cong \mathbb{Z}_{2}$ ), then $\mathcal{O}$ is one of the orbifolds in Figure 9 and 10. 


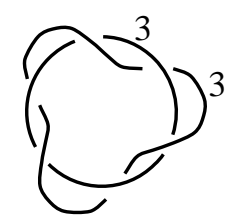

p.81 (type 2a)

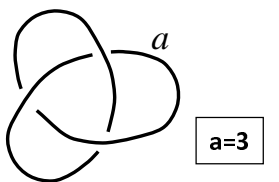

p.90 (type 2a)

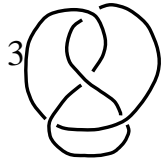

p.86 (type 4)

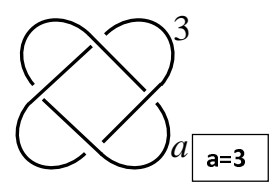

p.90 (type 2a)

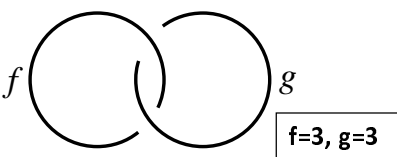

p.89 (type 2a)

Figure $8 . \mathcal{H}_{1}$ and $\mathcal{H}_{2}: \Gamma\left(\mathbb{Z}_{3}, 1, \mathbb{Z}_{3}\right)$

Proof. In this case the singular locus may be a link with two or three components or a knot. All components have index 2. In the case of a link with three components, each component is unknotted. In the case of a link with two components, one component is an unknot and the other component is a $(\leq 2)$-bridge knot. In the case of a knot, the singular locus is a $(\leq 3)$-bridge knot. Some cases of Montesinos graphs are also possible when the parameters of the tangles are all coprime (so the Montesinos graphs are really Montesinos links).

From now on, we will use the notation "the induced rational tangle" to denote the rational tangle which is obtained from a given tangled graph by substituting $(m / \operatorname{gcd}(m, n), n / \operatorname{gcd}(m, n))$ for $(m, n)$ where $(m, n)$ is a parameter of the given tangled graph and $m \neq 0$. If the tangled graph has a parameter $(0, n)$, then the induced rational tangle denotes just the remaining horizontal two strands after removing the vertical strut of index $n$. We will use the notation "The induced rational tangle is trivial." to denote that the parameter of the original tangled graph is $(0, n), n>1$. We will also use the notation "the induced Montesinos link" to denote the Montesinos link which is obtained from a given Montesinos graph by substituting the induced rational tangles for its tangled graphs. The induced Montesinos link is exactly the same as the Montesinos link which is obtained from the given Montesinos graph by removing all the struts.

Theorem 4.10. Suppose that $\mathcal{O}$ is non-hyperbolic and geometric. If both $\mathcal{H}_{1}$ and $\mathcal{H}_{2}$ are handlebody orbifolds modeled on $\Gamma\left(\mathbb{Z}_{2}, 1, \mathbb{D}_{2}\right)$ (so $\left.G \cong \mathbb{D}_{2}\right)$, then $\mathcal{O}$ is one of the orbifolds in Figure 11.

Proof. In this case, there are two vertices of degree 3 in the singular locus of $\mathcal{O}$ and the indices of the edge of the singular locus are all 2. If the singular locus is disconnected, then one component is unknotted and the other consists of two 


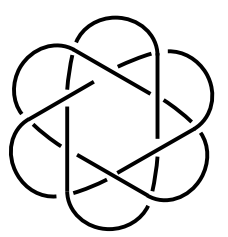

p.81 (type 2a)

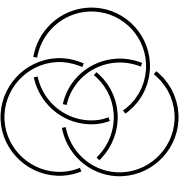

p.83 (type 2b)

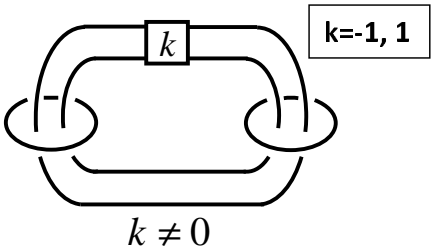

p.82 (type 2b)

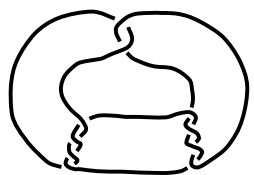

p.84 (type 2b)

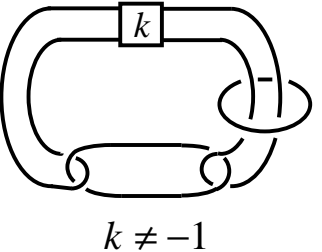

p.83 (type 2b)

Three components cases.

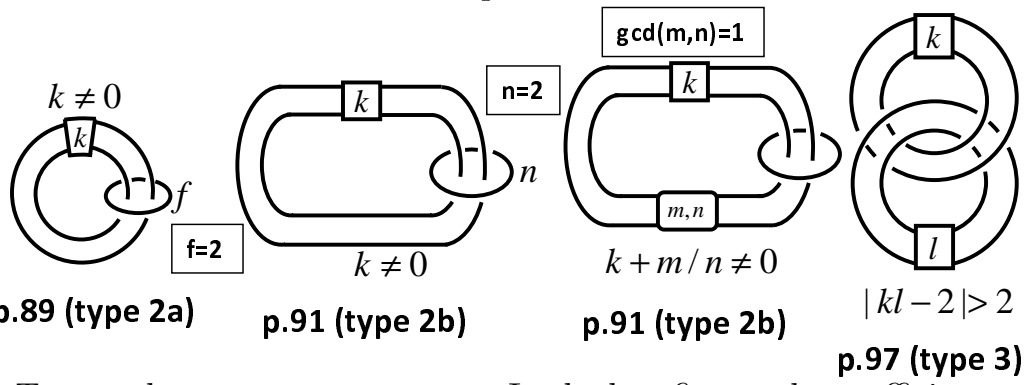

Two or three components cases. In the last figure, the coefficients $k, l$ need to be determined so that each component is a $(\leq 2)$ bridge knot and the number of components does not exceed 3 .

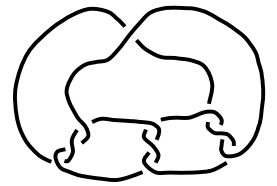

p.85 (type 2b)

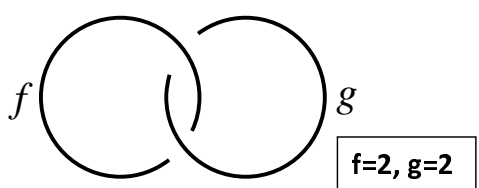

p.89 (type 2a)

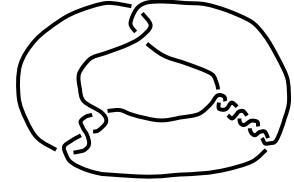

p.85 (type 2b)

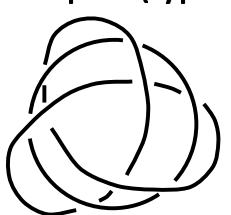

p.90 (type 2a)

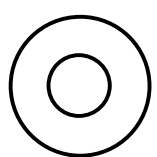

p.90 (type 2b)

Two components cases.

FiguRE $9 . \mathcal{H}_{1}$ and $\mathcal{H}_{2}: \Gamma\left(\mathbb{Z}_{2}, 1, \mathbb{Z}_{2}, 1, \mathbb{Z}_{2}\right)$ 


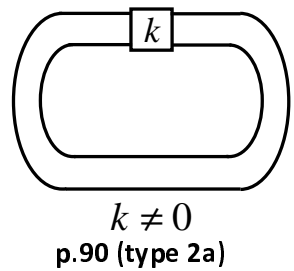

$\operatorname{gcd}\left(m_{1}, n\right)=1, \operatorname{gcd}\left(m_{2}, n\right)=1$

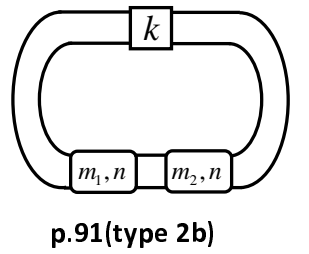

One or two components cases.
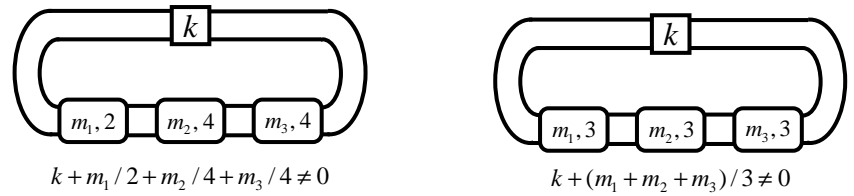

$k+m_{1} / 2+m_{2} / 4+m_{3} / 4 \neq 0$

p.84 (type 2b)

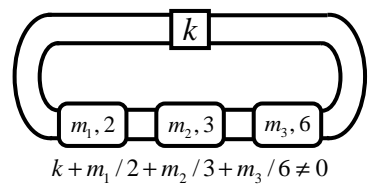

p.85 (type 2b)

p.85 (type 2b)

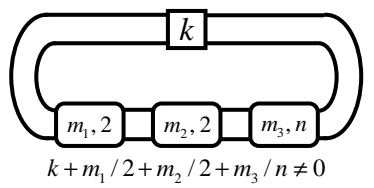

p.91 (type 2b)

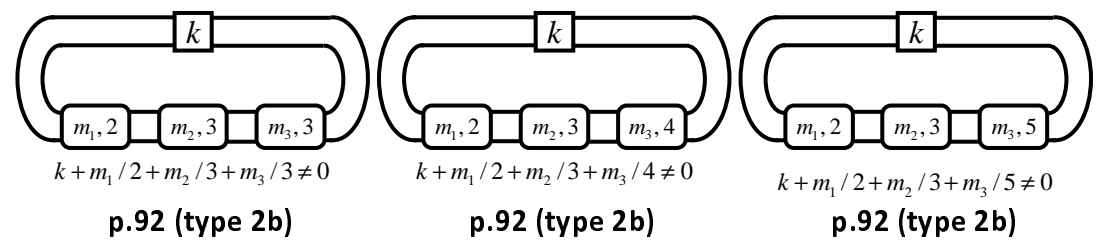

Montesinos graphs with three tangled graphs.

All tangles must have coprime coefficients.
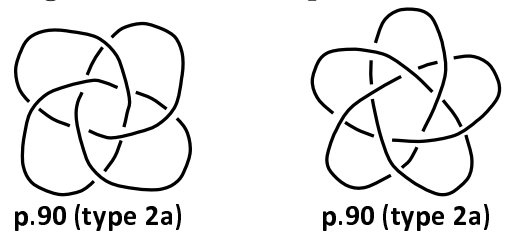

One component cases.

Figure $10 . \mathcal{H}_{1}$ and $\mathcal{H}_{2}: \Gamma\left(\mathbb{Z}_{2}, 1, \mathbb{Z}_{2}, 1, \mathbb{Z}_{2}\right)$

vertices of degree 3 and three edges which connect them (see Figure 12(a)). If the singular locus is connected, then it consists of two vertices of degree 3, an edge which connects them and two loops whose base points are the two vertices (see the top of Figure 12(b)), or it consists of two vertices of degree 3 and three 

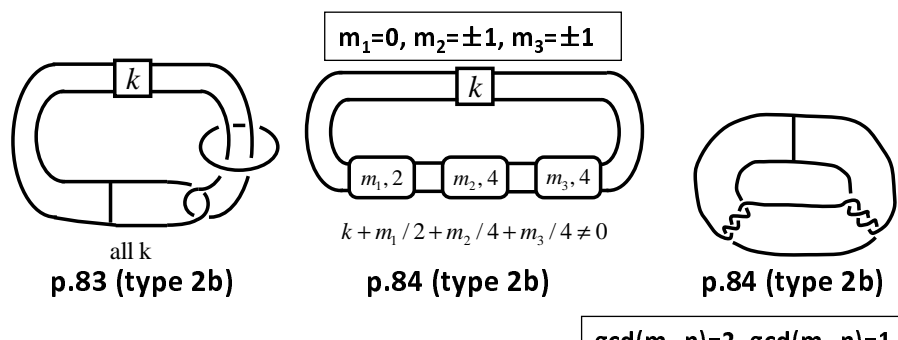

p.84 (type 2b)
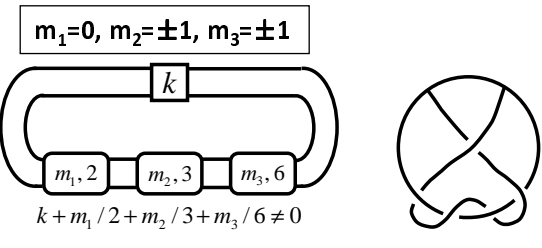

gcd $\left(m_{1}, n\right)=2, \operatorname{gcd}\left(m_{2}, n\right)=1$ or $\operatorname{gcd}\left(m_{1}, n\right)=2, \operatorname{gcd}\left(m_{2}, n\right)=1$

p.85 (type 2b) p.86 (type 4)

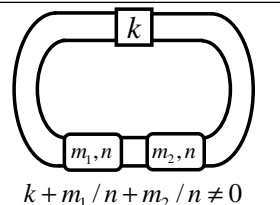

p.91(type 2b)

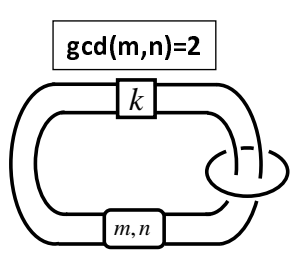

$k+m / n \neq 0$ p.91(type 2b)

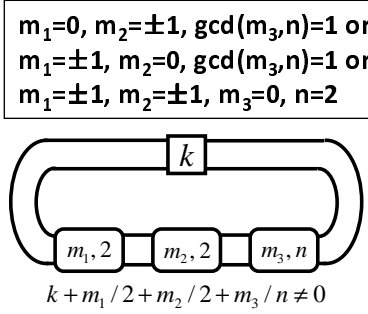
p.91 (type 2b)
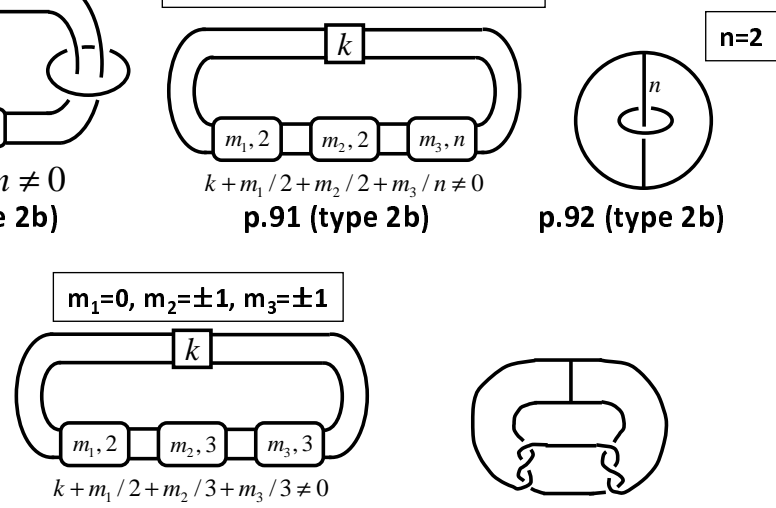

p.92 (type 2b)

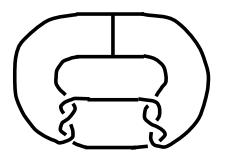
p.92 (type 2b)

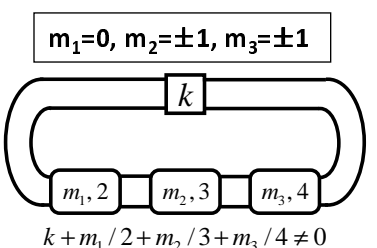

p.92 (type 2b)

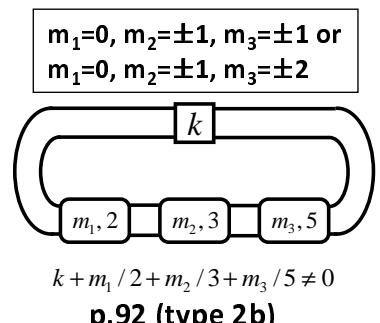

Figure $11 . \mathcal{H}_{1}$ and $\mathcal{H}_{2}: \Gamma\left(\mathbb{Z}_{2}, 1, \mathbb{D}_{2}\right)$. In the case of a Montesinos graph, the coefficients need to be determined so that the induced Montesinos link is a $(\leq 2)$-bridge link. 


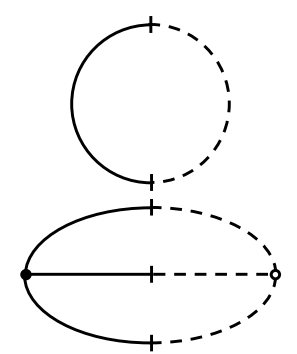

(a)

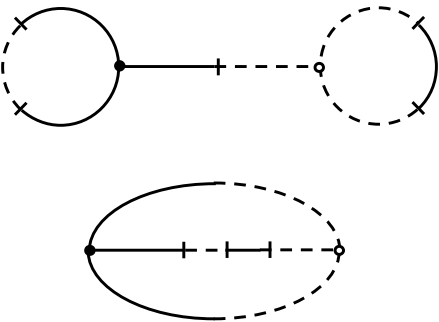

(b)

FiguRE 12. A rough sketch of singular loci in the cases of $\Gamma\left(\mathbb{Z}_{2}, 1, \mathbb{D}_{2}\right)$.

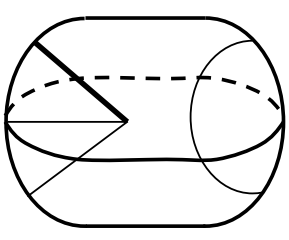

$\mathrm{H}_{1}$

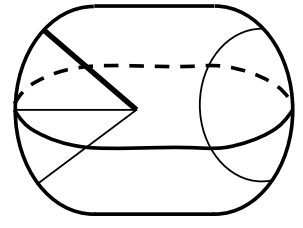

$\mathrm{H}_{2}$

Figure 13. The figure of the Claim of Theorem 4.10

edges which connect them (see bottom of Figure 12(b)). We need the following claim to consider the cases of Montesinos graphs.

Claim. Suppose that the singular locus is a Montesinos graph with three tangled graphs. Then $\left(m_{i}, n_{i}\right)=(0,2)$ for one $i$ and $\operatorname{gcd}\left(m_{i}, n_{i}\right)=1$ for the others. In addition, regardless of the number of the tangled graphs, the induced Montesinos link is a $(\leq 2)$-bridge link.

Proof of Claim. Suppose that the singular locus is a Montesinos graph. Since there are two vertices of degree 3 , one strut is from some tangled graph with non-coprime parameter and it connects both vertices of degree 3 . So the only possible case is as in Figure 13 (the strut is the union of two thick lines in the figure). If we remove the strut from the singular locus, then both $\mathcal{H}_{i}$ are rational tangle orbifolds. So the induced Montesinos link is a $(\leq 2)$-bridge link.

If all the induced rational tangles are non-trivial, then the induced Montesinos link has bridge number 3 by Lemma 4.4 , but this is a contradiction. So some induced rational tangle is trivial, i.e., $\left(m_{i}, n_{i}\right)=(0,2)$ for some $i$. This completes the proof of Claim.

Some cases of Montesinos graphs are possible. If the number of tangled graphs is 2 or less, then $\operatorname{gcd}\left(m_{i}, n_{i}\right)=2$ for only one $i$ and $\operatorname{gcd}\left(m_{i}, n_{i}\right)=1$ for the other. If the number of tangled graphs is 3 , then $\left(m_{i}, n_{i}\right)=(0,2)$ for one $i$ and $\operatorname{gcd}\left(m_{i}, n_{i}\right)=1$ for the others by Claim. 


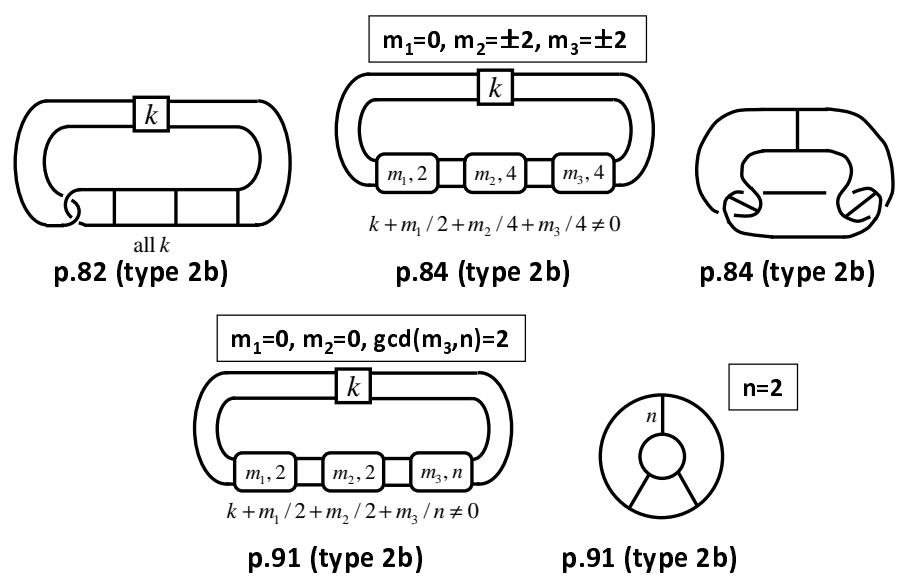

Figure $14 . \mathcal{H}_{1}$ and $\mathcal{H}_{2}: \Gamma\left(\mathbb{D}_{2}, 2, \mathbb{D}_{2}, 2, \mathbb{D}_{2}\right)$. In the case of a Montesinos graph, the coefficients need to be determined so that the induced Montesinos link is a $(\leq 2)$-bridge link.

Theorem 4.11. Suppose that $\mathcal{O}$ is non-hyperbolic and geometric. If both $\mathcal{H}_{1}$ and $\mathcal{H}_{2}$ are handlebody orbifolds modeled on $\Gamma\left(\mathbb{D}_{2}, 2, \mathbb{D}_{2}, 2, \mathbb{D}_{2}\right)$ (so $\left.G \cong \mathbb{D}_{2}\right)$, then $\mathcal{O}$ is one of the orbifolds in Figure 14.

Proof. In this case, the singular locus is connected and has six vertices, all of degree 3. All edges have index 2, and no edge separates or is a loop by itself. We need the following claim to consider the cases of Montesinos graphs.

Claim. Suppose that the singular locus is a Montesinos graph with three tangled graphs, as in Theorem 4.11. Then $\left(m_{i}, n_{i}\right)=(0,2)$ for one $i$ and $\operatorname{gcd}\left(m_{i}, n_{i}\right)=$ 2 for the others. Moreover, the induced Montesinos link is a $(\leq 2)$-bridge link.

Proof of Claim. Suppose that the singular locus is a Montesinos graph. Since there are six vertices of degree 3 , three struts of index 2 are from three tangled graphs with non-coprime parameters. So $\operatorname{gcd}\left(m_{i}, n_{i}\right)=2$ for all $i=1,2,3$. It is easy to see that no two struts can be adjacent. We will consider each possible case.

Case 1. Consider the case when no strut is entirely contained in some $\mathcal{H}_{i}$ (see Figure 15 (a)). If we remove the three struts from the singular locus, then the result $L$ consists of two arcs each of which is trivially embedded in $\mathcal{H}_{i}$, $i=1,2$, so $L$ is an unknot. If all the induced rational tangles are non-trivial, then $b(L)=3$ by Lemma 4.4 , a contradiction. Hence some induced rational tangle is trivial, i.e., $\left(m_{i}, n_{i}\right)=(0,2)$ for some $i$.

Case 2. Consider the case when some strut $e_{1}$ is entirely contained in one of the $\mathcal{H}_{i}$, say $\mathcal{H}_{1}$. As shown in Figure 15(b), some strut $e_{2}$ must be entirely contained in $\mathcal{H}_{2}$, since otherwise a strut would be adjacent to $e_{1}$ in $\mathcal{H}_{1}$. So 

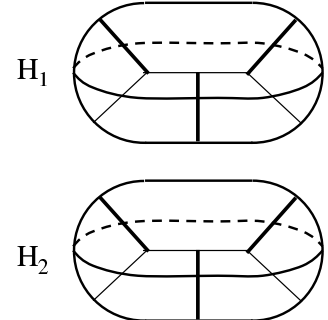

(a)
$\mathrm{H}_{1}$

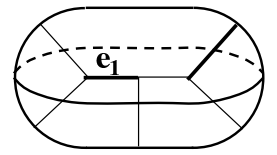

$\mathrm{H}_{2}$

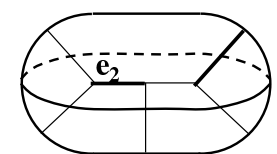

(b)

Figure 15. The figure of the Claim of Theorem 4.11

removing all three struts from the singular locus produces a rational tangle orbifold, so the remaining singular locus $L$ is a $(\leq 2)$-bridge link and is the induced Montesinos link. If the induced rational tangles are all non-trivial, then $b(L)=3$ by Lemma 4.4, a contradiction. Hence some induced rational tangle is trivial, i.e., $\left(m_{i}, n_{i}\right)=(0,2)$ for some $i$. This completes the proof of the Claim.

Some cases of Montesinos graphs are possible. Since the number of struts is 3 , we only need to check the cases of Montesinos graphs with three tangled graphs and we can say that $\left(m_{i}, n_{i}\right)=(0,2)$ for one $i$ and $\operatorname{gcd}\left(m_{i}, n_{i}\right)=2$ for the others by the Claim.

Theorem 4.12. Suppose that $\mathcal{O}$ is non-hyperbolic and geometric. If $\mathcal{H}_{1}$ and $\mathcal{H}_{2}$ are handlebody orbifolds modeled on $\Gamma\left(\mathbb{Z}_{2}, 1, \mathbb{D}_{2}\right)$ and $\Gamma\left(\mathbb{D}_{2}, 2, \mathbb{D}_{2}, 2, \mathbb{D}_{2}\right)$ (so $\left.G \cong \mathbb{D}_{2}\right)$, then $\mathcal{O}$ is one of the orbifolds in Figure 16 .

Proof. The singular locus is a connected graph with four vertices, all of degree 3 , and the indices of all edges are 2 . We need the following claim to consider the cases of Montesinos graphs.

Claim. If the singular locus is a Montesinos graph with three tangled graphs in the configuration of Theorem 4.12, then $\left(m_{i}, n_{i}\right)=(0,2)$ for one $i, \operatorname{gcd}\left(m_{i}, n_{i}\right)$ $=2$ for another $i$ and $\operatorname{gcd}\left(m_{i}, n_{i}\right)=1$ for the other $i$. In addition, regardless of the number of the tangled graphs, the induced Montesinos link is a $(\leq 2)$-bridge link.

Poof of Claim. Suppose that the singular locus is a Montesinos graph with three tangled graphs. Since there are four vertices of degree 3, two struts are from two tangled graphs with non-coprime parameters. So $\operatorname{gcd}\left(m_{i}, n_{i}\right)=2$ for two $i$ 's and $\operatorname{gcd}\left(m_{i}, n_{i}\right)=1$ for the other. We will consider each possible case.

Case 1. Suppose that a strut is entirely in $\mathcal{H}_{2}$. Since the other strut meets the vertex of degree 3 in $\mathcal{H}_{1}$, the only possible situation is as in Figure $17(\mathrm{a})$. If we remove two struts from the singular locus then both $\mathcal{H}_{1}$ and $\mathcal{H}_{2}$ are rational tangle orbifolds, so the induced Montesinos link is a $(\leq 2)$-bridge link. 


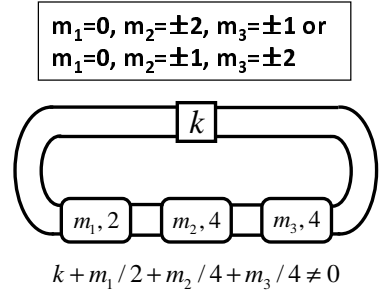

p.84 (type 2b)

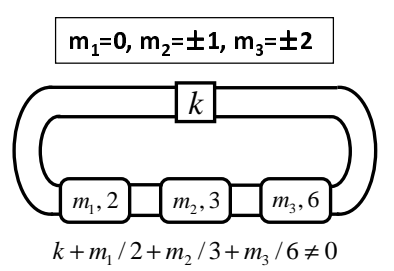

p.85 (type 2b)

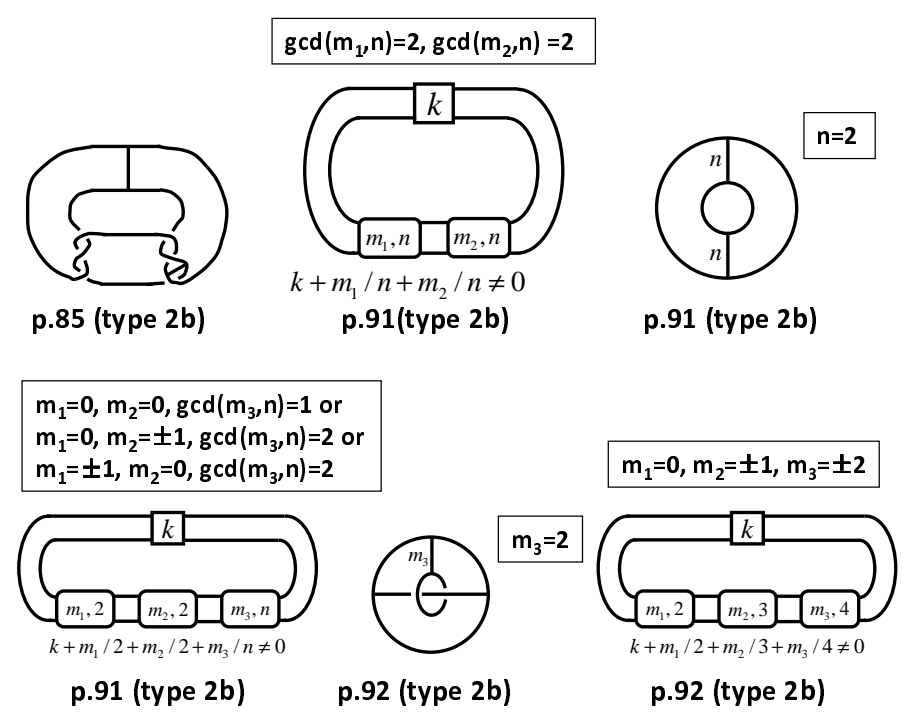

Figure 16 . $\mathcal{H}_{1}: \Gamma\left(\mathbb{Z}_{2}, 1, \mathbb{D}_{2}\right), \mathcal{H}_{2}: \Gamma\left(\mathbb{D}_{2}, 2, \mathbb{D}_{2}, 2, \mathbb{D}_{2}\right)$. In the case of a Montesinos graph, the coefficients must be determined so that the induced Montesinos link is a $(\leq 2)$-bridge link.

By Lemma 4.4 the number of non-trivial induced rational tangles is less than or equal to 2 . So $\left(m_{i}, n_{i}\right)=(0,2)$ for some $i$.

Case 2. Suppose that no strut is entirely contained in $\mathcal{H}_{2}$. We must have the configuration of Figure 17(b), so removing the struts from the singular locus produces a trivial knot. Lemma 4.4 now shows that some induced rational tangle is trivial, i.e., $\left(m_{i}, n_{i}\right)=(0,2)$ for some $i$. This completes the proof of the Claim.

Some cases of Montesinos graphs with at least two tangled graphs are possible. If the number of tangled graphs is 2 , then $\operatorname{gcd}\left(m_{i}, n_{i}\right)=2$ for all $i$. If the number of tangled graphs is 3 , then $\left(m_{i}, n_{i}\right)=(0,2)$ for one $i, \operatorname{gcd}\left(m_{i}, n_{i}\right)=2$ for another $i$ and $\operatorname{gcd}\left(m_{i}, n_{i}\right)=1$ for the other $i$ by the Claim. The induced Montesinos link is a $(\leq 2)$-bridge link by the Claim. 


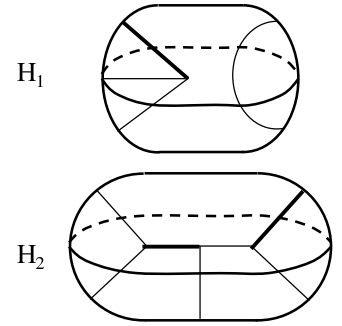

(a)
$\mathrm{H}_{1}$

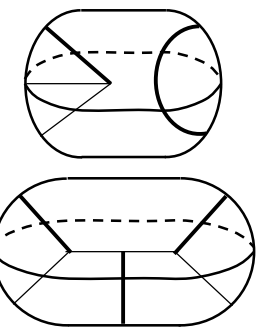

(b)

Figure 17. The figure of the Claim of Theorem 4.12

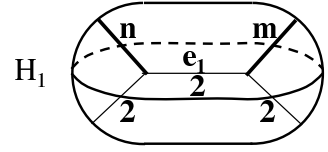

$\mathrm{H}_{2}$

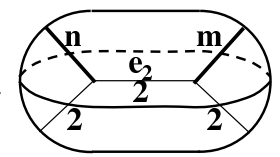

Figure 18. Lemma 4.13

Lemma 4.13. Suppose that $\mathcal{O}$ consists of two handlebody orbifolds $\mathcal{H}_{1}$ and $\mathcal{H}_{2}$ modeled on both $\Gamma\left(\mathbb{D}_{3}, 2, \mathbb{D}_{3}\right)$ or both $\Gamma\left(\mathbb{D}_{2}, 2, \mathbb{D}_{4}\right)$ or both $\Gamma\left(\mathbb{D}_{2}, 2, \mathbb{D}_{3}\right)$. Suppose that the singular locus is a Montesinos graph. Then the induced Montesinos link is an unknot. If the number of tangled graphs is 3 , then for some $i$,

$$
\left(m_{i}, n_{i}\right)=(0, k) \text { where } \begin{cases}k=3, & \text { if } G \cong \mathbb{D}_{3}, \\ k=2 \text { or } 4, & \text { if } G \cong \mathbb{D}_{4}, \\ k=2 \text { or } 3 . & \text { if } G \cong \mathbb{D}_{6} .\end{cases}
$$

Proof. The singular locus in $\mathcal{O}$ is a connected graph with four vertices of degree 3. Suppose that it is a Montesinos graph. Then two struts are from two tangled graphs with non-coprime parameters. So the number of tangled graphs is at least 2. It is easy to see that the two struts cannot be adjacent. Let the only edge which is contained entirely in each $\mathcal{H}_{i}$ be $e_{i}$. Since any edge whose index is greater than 2 must be among the two struts, no $e_{i}$ can be among the struts in this Montesinos graph (see Figure 18). In this configuration, if we remove the two struts from the singular locus then the induced Montesinos link is an unknot. So if the number of tangled graphs is 3 , then some induced rational tangle is trivial by Lemma 4.4. So we get $\left(m_{i}, n_{i}\right)=(0, k)$ where $k$ is determined by $G$.

Theorem 4.14. Suppose that $\mathcal{O}$ is non-hyperbolic and geometric. If both $\mathcal{H}_{1}$ and $\mathcal{H}_{2}$ are handlebody orbifolds modeled on $\Gamma\left(\mathbb{D}_{3}, 2, \mathbb{D}_{3}\right)$ (so $\left.G \cong \mathbb{D}_{3}\right)$, then $\mathcal{O}$ is the orbifold in Figure 19. 


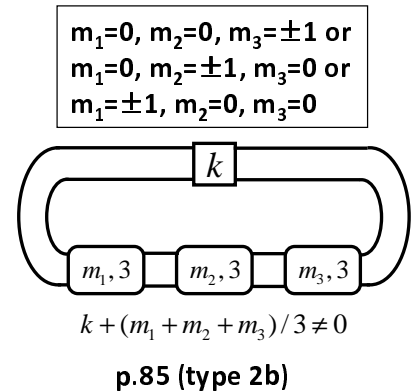

$\operatorname{gcd}\left(m_{1}, n\right)=3, \operatorname{gcd}\left(m_{2}, n\right)=3$

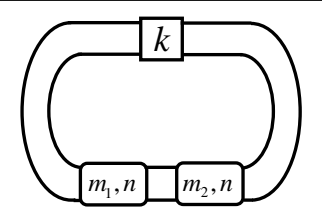

$k+m_{1} / n+m_{2} / n \neq 0$

p.91(type 2b)

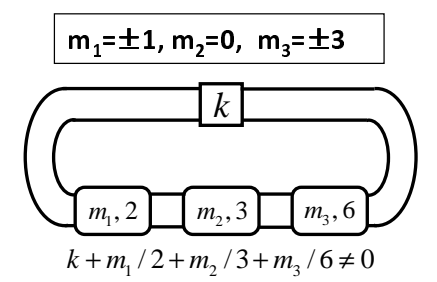

p.85 (type 2b)

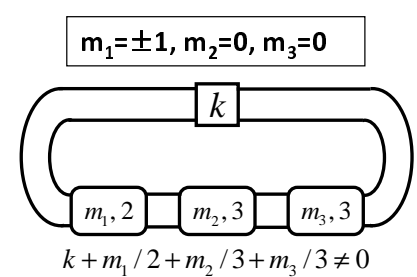

p.92 (type 2b)

Figure 19 . $\mathcal{H}_{1}$ and $\mathcal{H}_{2}: \Gamma\left(\mathbb{D}_{3}, 2, \mathbb{D}_{3}\right)$. In the case of a Montesinos graph, the coefficients must be determined so that the induced Montesinos link is an unknot.

Proof. In this case, the singular locus is a connected graph with four vertices, all of degree 3, and two edges of index 3. All other edges have index 2. No edge separates or is a loop, and the two edges of index 3 cannot be adjacent.

Some orbifolds from Montesinos graphs with at least two tangled graphs are possible. If the number of tangled graphs of a given Montesinos graph is 2, then $\operatorname{gcd}\left(m_{i}, n_{i}\right)=3$ for all $i$. If the number of tangled graphs of a given Montesinos graph is 3 , then $\left(m_{i}, n_{i}\right)=(0,3)$ for one $i$ by Lemma $4.13, \operatorname{gcd}\left(m_{i}, n_{i}\right)=3$ for another $i$ and $\operatorname{gcd}\left(m_{i}, n_{i}\right)=1$ for the other $i$. The induced Montesinos link is an unknot by Lemma 4.13 .

Theorem 4.15. Suppose that $\mathcal{O}$ is non-hyperbolic and geometric. If both $\mathcal{H}_{1}$ and $\mathcal{H}_{2}$ are handlebody orbifolds modeled on $\Gamma\left(\mathbb{D}_{2}, 2, \mathbb{D}_{4}\right)\left(\right.$ so $\left.G \cong \mathbb{D}_{4}\right)$, then $\mathcal{O}$ is one of the orbifolds in Figure 20.

Proof. In this case, the singular locus is a connected graph with four vertices, all of degree 3 , and with one edge of index 4 . All other edges have index 2. No edge separates or is a loop.

Some orbifolds from Montesinos graphs with at least two tangled graphs are possible. If the number of tangled graphs of a given Montesinos graph is 2, then $\operatorname{gcd}\left(m_{i}, n_{i}\right)=2$ for one $i$ and $\operatorname{gcd}\left(m_{i}, n_{i}\right)=4$ for the other $i$. If the number of tangled graphs of a given Montesinos graph is 3 , then $\left(m_{i}, n_{i}\right)=(0,2)$ for 


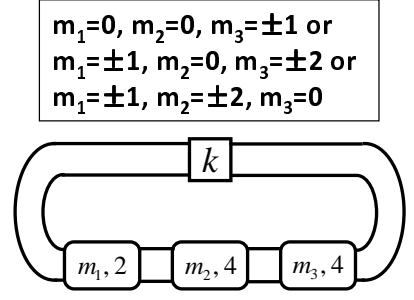

$k+m_{1} / 2+m_{2} / 4+m_{3} / 4 \neq 0$

p.84 (type 2b)
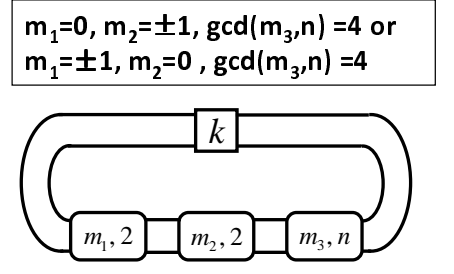

$k+m_{1} / 2+m_{2} / 2+m_{3} / n \neq 0$

p.91 (type 2b)

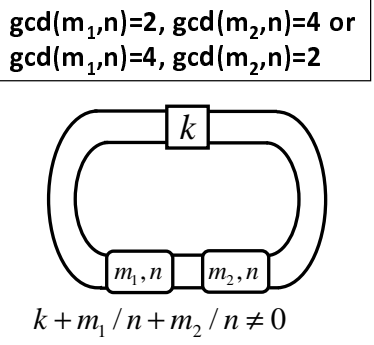

p.91(type 2b)
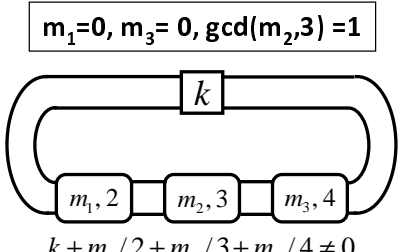

p.92 (type 2b)

Figure 20. $\mathcal{H}_{1}$ and $\mathcal{H}_{2}: \Gamma\left(\mathbb{D}_{2}, 2, \mathbb{D}_{4}\right)$. In the case of a Montesinos graph, the coefficients must be determined so that the induced Montesinos link is an unknot.

one $i$ by Lemma 4.13, $\operatorname{gcd}\left(m_{i}, n_{i}\right)=4$ for another $i$ and $\operatorname{gcd}\left(m_{i}, n_{i}\right)=1$ for the other $i$ or $\left(m_{i}, n_{i}\right)=(0,4)$ for one $i$ by Lemma $4.13, \operatorname{gcd}\left(m_{i}, n_{i}\right)=2$ for another $i$ and $\operatorname{gcd}\left(m_{i}, n_{i}\right)=1$ for the other $i$. The induced Montesinos link is an unknot by Lemma 4.13 .

Theorem 4.16. Suppose that $\mathcal{O}$ is non-hyperbolic and geometric. If both $\mathcal{H}_{1}$ and $\mathcal{H}_{2}$ are handlebody orbifolds modeled on $\Gamma\left(\mathbb{D}_{2}, 2, \mathbb{D}_{3}\right)$ (so $\left.G \cong \mathbb{D}_{6}\right)$, then $\mathcal{O}$ is one of the orbifolds in Figure 21.

Proof. In this case, the singular locus is a connected graph with four vertices, all of degree 3 , and with one edge of index 3 . All other edges have index 2 . No edge separates or is a loop.

Some orbifolds from Montesinos graphs with at least two tangled graphs are possible. If the number of tangled graphs of given Montesinos graph is 2 , then $\operatorname{gcd}\left(m_{i}, n_{i}\right)=2$ for one $i$ and $\operatorname{gcd}\left(m_{i}, n_{i}\right)=3$ for the other $i$. If the number of tangled graphs of given Montesinos graph is 3 , then $\left(m_{i}, n_{i}\right)=(0,2)$ for one $i$ by Lemma 4.13, $\operatorname{gcd}\left(m_{i}, n_{i}\right)=3$ for another $i$ and $\operatorname{gcd}\left(m_{i}, n_{i}\right)=1$ for the other $i$ or $\left(m_{i}, n_{i}\right)=(0,3)$ for one $i$ by Lemma $4.13, \operatorname{gcd}\left(m_{i}, n_{i}\right)=2$ for another $i$ and $\operatorname{gcd}\left(m_{i}, n_{i}\right)=1$ for the other $i$. The induced Montesinos link is an unknot by Lemma 4.13 . 


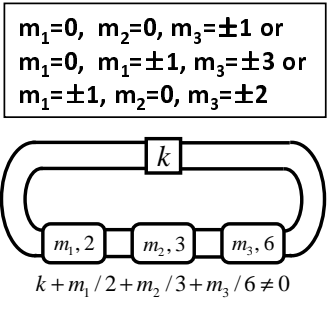

p.85 (type 2b)

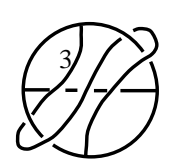

p.86 (type 4) $\operatorname{gcd}\left(m_{1}, n\right)=2, \operatorname{gcd}\left(m_{2}, n\right)=3$ or

$\operatorname{gcd}\left(m_{1}, n\right)=3, \operatorname{gcd}\left(m_{2}, n\right)=2$

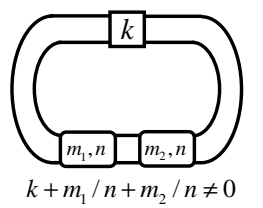

p.91(type 2b)
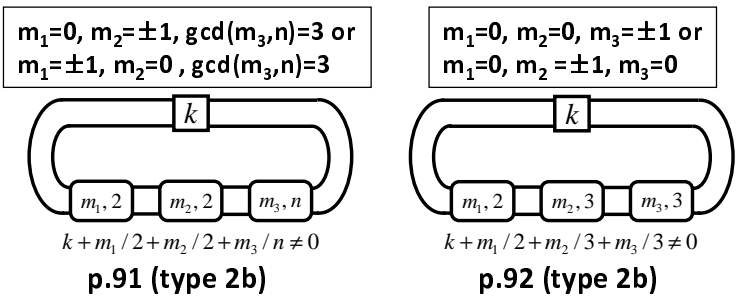

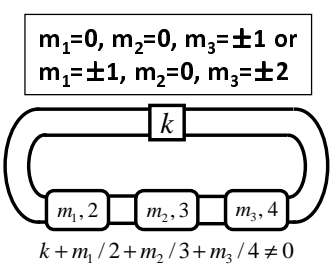

p.92 (type 2b) p.92 (type 2b)

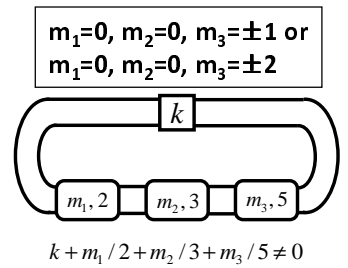

p.92 (type 2b)

Figure 21. $\mathcal{H}_{1}$ and $\mathcal{H}_{2}: \Gamma\left(\mathbb{D}_{2}, 2, \mathbb{D}_{3}\right)$. In the case of a Montesinos graph, the coefficients must be determined so that the induced Montesinos link is an unknot.

In the remaining part of this section, we will consider the cases when the underlying space of $\mathcal{O}^{3}$ is $L(p, q)$.

Definition $4.17([4])$. Let $V$ be a solid torus and let $a$ be an arc properly embedded in $V$. We say that $a$ is trivially embedded in $V$ if there is a disk $D$ in $V$ such that $D \cap \partial V$ is an arc $b$ and $\operatorname{cl}(\partial D-b)=a$. Let $K$ be a knot in a lens space. We say that $K$ is a one bridge knot in a lens space if there is a Heegaard splitting $\left(V_{1}, V_{2} ; F\right)$ of the lens space such that each $V_{i} \cap K$ is an arc trivially embedded in $V_{i}$ for $i=1,2$.

Theorem 4.18. If both $\mathcal{H}_{1}$ and $\mathcal{H}_{2}$ are handlebody orbifolds modeled on $\Gamma\left(\mathbb{Z}_{2}\right.$, 1) $\left(\right.$ so $\left.G \cong \mathbb{Z}_{2}\right)$, then the underlying space is homeomorphic to $L(p, q)$ and the singular locus is a one bridge knot in $L(p, q)$. Moreover, if the underlying space is homeomorphic to $\mathbb{S}^{3}$ and $\mathcal{O}$ is non-hyperbolic and geometric, then the singular locus is an unknot (Figure 22 means the existence of such $\mathcal{O}$ ). 


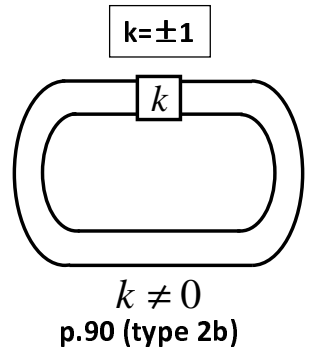

FiguRE 22. $\mathcal{H}_{1}$ and $\mathcal{H}_{2}: \Gamma\left(\mathbb{Z}_{2}, 1\right)$. A case of underlying space $\mathbb{S}^{3}$. The coefficient is determined so that the Montesinos graph is an unknot.

Proof. By Lemma 4.2 the underlying space is $L(p, q)$. Because the singular locus in each $\mathcal{H}_{i}$ for $i=1,2$ is a trivially embedded arc of index 2 , the singular locus of $\mathcal{O}$ is a one bridge knot in a lens space of index 2 .

If the underlying space is homeomorphic to $\mathbb{S}^{3}$, then the singular locus is just an unknot. So we only need to find a candidate which induce an unknot of index 2 as a singular locus in Dunbar's figures.

Theorem 4.19. If both $\mathcal{H}_{1}$ and $\mathcal{H}_{2}$ are handlebody orbifolds modeled on $\Gamma\left(\mathbb{Z}_{2}\right.$, $1,\left(\mathbb{Z}_{2}, 2\right)$ ) (so $\left.G \cong \mathbb{Z}_{2}\right)$, then the underlying space is homeomorphic to $L(p, q)$. The singular locus consists of two core loops of $\mathcal{H}_{i}$ and a one bridge knot in lens space. If the underlying space is homeomorphic to $\mathbb{S}^{3}$ and $\mathcal{O}$ is non-hyperbolic and geometric, then $\mathcal{O}$ is one of the orbifolds in Figure 23.

Proof. By Lemma 4.2 the underlying space is $L(p, q)$. The singular locus in $\mathcal{O}$ is easily deduced from the singular loci in the $\mathcal{H}_{i}$, and it consists of three loops of index 2.

From now on we consider the cases of the underlying space $\mathbb{S}^{3} \cong L(1, q)$. Both two core loops in $\mathcal{H}_{i}$ for $i=1,2$ are unknots and the linking number is \pm 1 . This rules out many candidates in Dunbar's figures. Moreover, the union of two arcs from each $\mathcal{H}_{i}$ for $i=1,2$ is an unknot from the construction of $L(1, q)$ (but it can be linked with the core of some $\mathcal{H}_{i}$ ). So we only need to find figures with three unknotted components, two of them linked with linking number \pm 1 .

Theorem 4.20. If $\mathcal{H}_{1}$ and $\mathcal{H}_{2}$ are handlebody orbifolds modeled on $\Gamma\left(\mathbb{Z}_{2}, 1\right)$ and $\Gamma\left(\mathbb{Z}_{2}, 1,\left(\mathbb{Z}_{2}, 2\right)\right.$ ) (so $\left.G \cong \mathbb{Z}_{2}\right)$, then the underlying space is homeomorphic to $L(p, q)$. The singular locus consists of the core of $\mathcal{H}_{2}$ and a one bridge knot in lens space. If the underlying space is homeomorphic to $\mathbb{S}^{3}$ and $\mathcal{O}$ is non-hyperbolic and geometric, then $\mathcal{O}$ is one of the orbifolds in Figure 24. 


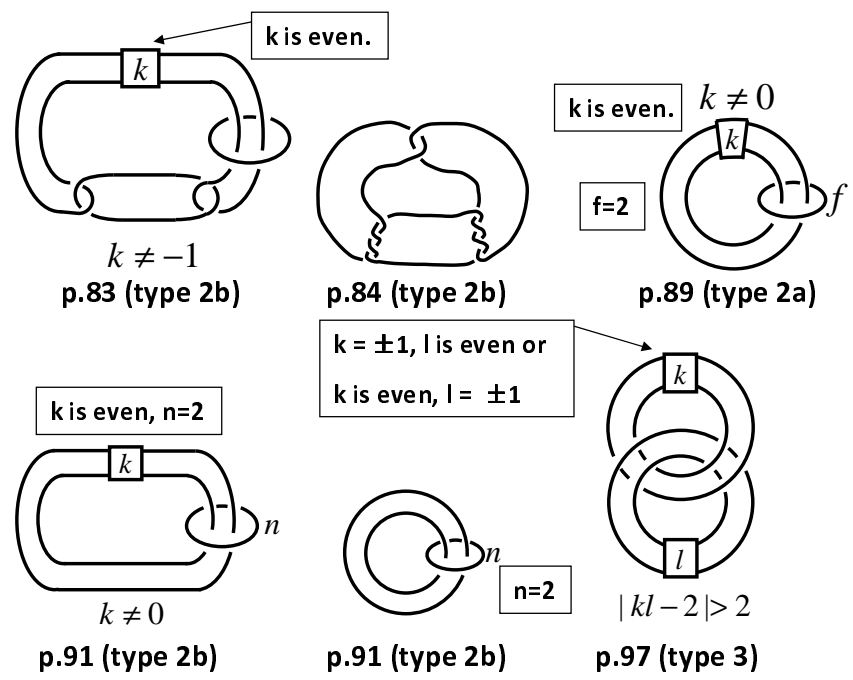

FiguRE 23. $\mathcal{H}_{1}$ and $\mathcal{H}_{2}: \Gamma\left(\mathbb{Z}_{2}, 1,\left(\mathbb{Z}_{2}, 2\right)\right)$. The cases of underlying space $\mathbb{S}^{3}$. In addition, the 3rd figure in "two or three components cases" in Figure 9 and the figures in "Montesinos graphs with three tangled graphs" in Figure 10 are also possible if the coefficients are determined so that the components are three unknotted loops and two of them have linking number \pm 1 .

Proof. By Lemma 4.2 the underlying space is $L(p, q)$. The singular locus in $\mathcal{O}$ is easily deduced from the singular loci in the $\mathcal{H}_{i}$ for $i=1,2$, it consists of two loops of index 2 .

From now on we consider the cases of the underlying space $\mathbb{S}^{3} \cong L(1, q)$. The core loop in $\mathcal{H}_{2}$ is an unknot. Moreover, the union of two arcs from each $\mathcal{H}_{i}$ for $i=1,2$ is an unknot from the construction of $L(1, q)$ (but it can be linked with the core of $\mathcal{H}_{2}$ ). So we only need to find figures with two unknotted components.

Theorem 4.21. If both $\mathcal{H}_{1}$ and $\mathcal{H}_{2}$ are handlebody orbifolds modeled on $\Gamma\left(\mathbb{D}_{2}\right.$, 2) (so $G \cong \mathbb{D}_{2}$ ), then the underlying space is homeomorphic to $L(p, q)$. The singular locus consists of two core loops in $\mathcal{H}_{i}$ and an edge connecting the two loops. If the underlying space is homeomorphic to $\mathbb{S}^{3}$ and $\mathcal{O}$ is non-hyperbolic and geometric, then $\mathcal{O}$ is one of the orbifolds in Figure 25.

Proof. By Lemma 4.2 the underlying space is $L(p, q)$. The indices of edges in the singular locus are all 2 . The singular locus in $\mathcal{O}$ is easily deduced from the singular loci in the $\mathcal{H}_{i}$. It consists of two vertices of degree 3 , a loop based on each vertex, and an edge connecting the vertices. 


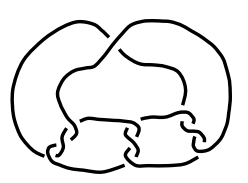

p.85 (type 2b)

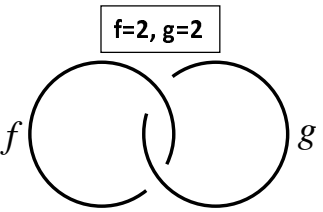

p. 89 (type 2a)

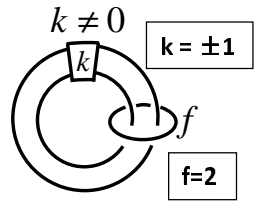

p. 89 (type 2a)

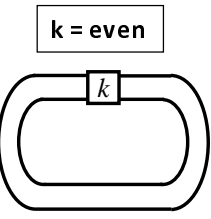

$k \neq 0$ p.90 (type 2b)

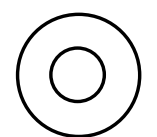

p.90 (type 2b)

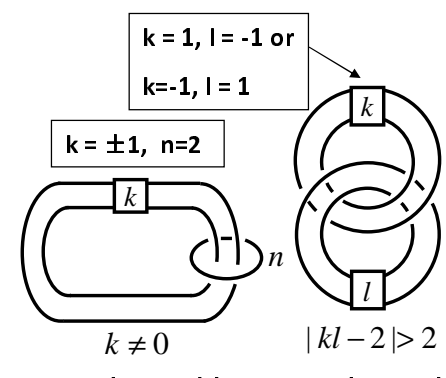

p.91 (type 2b)

p.97 (type 3)

Figure 24. $\mathcal{H}_{1}: \Gamma\left(\mathbb{Z}_{2}, 1\right)$ and $\mathcal{H}_{2}: \Gamma\left(\mathbb{Z}_{2}, 1,\left(\mathbb{Z}_{2}, 2\right)\right)$. The cases of underlying space $\mathbb{S}^{3}$. In addition, the 3 rd figure in "two or three components cases" in Figure 9, the 2nd figure in "one or two components cases" in Figure 10 and the figures in "Montesinos graphs with three tangled graphs" in Figure 10 are also possible if the coefficients are determined so that the components are two unknotted loops.

From now on we consider the cases whose underlying spaces are $\mathbb{S}^{3}$.

Suppose that the singular locus is a Montesinos graph. Since there are only two vertices of degree 3, exactly one strut is from some tangled graph. If we remove the strut, then the remaining singular locus is a link with two components. So we can say that the number of components of the induced Montesinos link is 2. In addition, the strut must connect two different components. This rules out most Montesinos graphs as possible candidates. For example, consider the Montesinos graph $m\left(0 \mid k ;\left(m_{1}, 2\right),\left(m_{2}, 4\right),\left(m_{3}, 4\right)\right)$ (see p. 84 of [2]. The parameter $(m, n)$ of the tangled graph uses the continued fraction of $m / n$ by Dunbar's notation). Possible triples of parameters $\left(m_{1}, m_{2}, m_{3}\right)$ are $(0, \pm 1, \pm 1)$, $( \pm 1, \pm 2, \pm 1)$ and $( \pm 1, \pm 1, \pm 2)$. In the first triples, the strut connects the same component. In the second and third triples, the strut connects different components, but the number of components of the induced Montesinos link is 3 . So the Montesinos graph with parameters $m\left(0 \mid k ;\left(m_{1}, 2\right),\left(m_{2}, 4\right),\left(m_{3}, 4\right)\right)$ is impossible. In the case of the Montesinos graph $m\left(0 \mid k ;\left(m_{1}, 2\right),\left(m_{2}, 2\right),\left(m_{3}, n\right)\right)$, (see p. 91 of [2]) possible triples of parameters $\left(m_{1}, m_{2}, m_{3}\right)$ are $\left(0, \pm 1, \pm m_{3}\right)$ with $\operatorname{gcd}\left(m_{3}, n\right)=1,\left( \pm 1,0, \pm m_{3}\right)$ with $\operatorname{gcd}\left(m_{3}, n\right)=1$ and $\left( \pm 1, \pm 1, \pm m_{3}\right)$ with $\operatorname{gcd}\left(m_{3}, n\right)=2$. In the first and second triples, the strut is attached to 


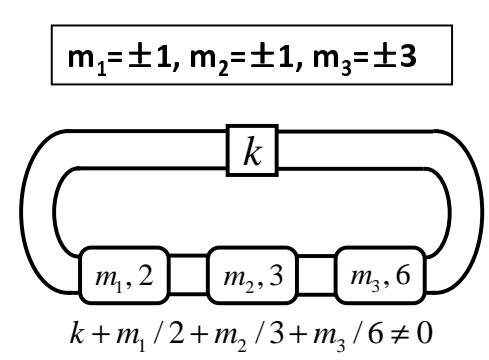

p.85 (type 2b)

$m_{1}=0, m_{2}= \pm 1, m_{3}= \pm 1, k$ is even

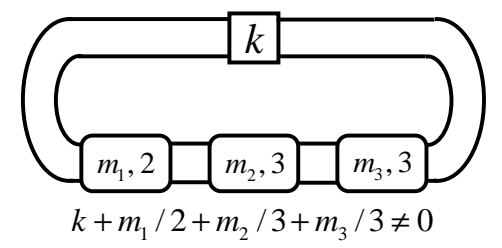

p.92 (type 2b)

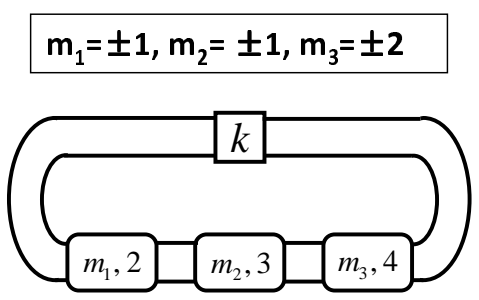

$k+m_{1} / 2+m_{2} / 3+m_{3} / 4 \neq 0$

p.92 (type 2b)

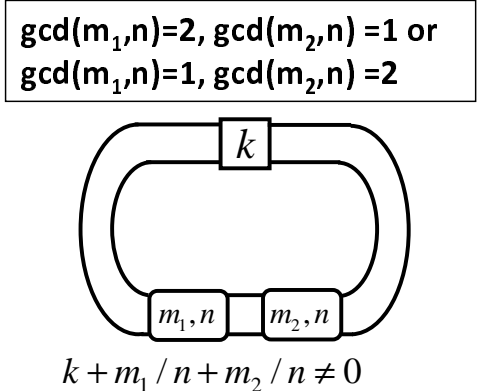

p.91(type 2b)

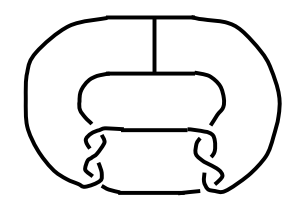

p.92 (type 2b)
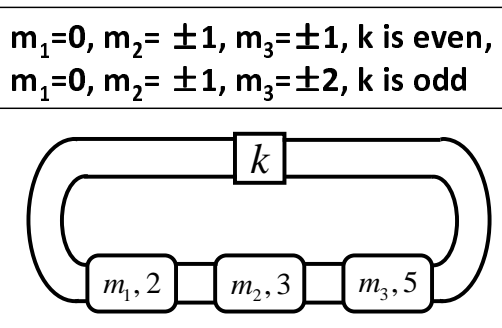

$k+m_{1} / 2+m_{2} / 3+m_{3} / 5 \neq 0$

p.92 (type 2b)

Figure 25. $\mathcal{H}_{1}$ and $\mathcal{H}_{2}: \Gamma\left(\mathbb{D}_{2}, 2\right)$. The cases of underlying space $\mathbb{S}^{3}$. In the second figure, the coefficients must be determined so that the number of components of the induced Montesinos link is 2 .

the same component. In the third, there is a component that does not meet the strut. So the Montesinos graph $m\left(0 \mid k ;\left(m_{1}, 2\right),\left(m_{2}, 2\right),\left(m_{3}, n\right)\right)$ is impossible.

In some cases we can restrict the coefficient $k$ of the Montesinos graphs. For example, consider the Montesinos graph $m\left(0 \mid k ;\left(m_{1}, 2\right),\left(m_{2}, 3\right),\left(m_{3}, 3\right)\right)$ (see p. 92 in [2]). Possible triples of parameters $\left(m_{1}, m_{2}, m_{3}\right)$ are $(0, \pm 1, \pm 1)$. In this case, if $k$ is odd, then the number of components of the induced Montesinos link is 1 and if $k$ is even, then the number of components of induced Montesinos 
link is 2 and the strut connects different components. So we can say that if this case occurs, then $k$ must be even.

\section{Some hyperbolic quotients of $G$-manifolds of genus two are impossible.}

In this section, we will prove that the fundamental groups of the quotient orbifolds are not in a certain class $\mathcal{D}$.

The class $\mathcal{D}$ was defined by E. Klimenko and N. Kopteva [3], who classified the orbifolds whose fundamental groups belong to a certain class $\mathcal{R} \mathcal{P}$. A pair $(f, g)$ of elements in $\operatorname{PSL}(2, \mathbb{C})$ is called an $\mathcal{R} \mathcal{P}$-pair if $\operatorname{tr}^{2}(f), \operatorname{tr}^{2}(g)$, and $\operatorname{tr}([f, g])$ are all real. The class $\mathcal{R} \mathcal{P}$ of Kleinian groups is defined by

$$
\mathcal{R} \mathcal{P}=\{\Gamma: \Gamma=\langle f, g\rangle \text { for some } \mathcal{R} \mathcal{P} \text {-pair }(f, g)\}
$$

For an $\mathcal{R} \mathcal{P}$-pair $(f, g)$, define $\beta=\operatorname{tr}^{2}(f)-4, \beta^{\prime}=\operatorname{tr}^{2}(g)-4$, and $\gamma=$ $\operatorname{tr}([f, g])-2$. Define a subclass of $\mathcal{R} \mathcal{P}$ by

$$
\begin{aligned}
& \mathcal{D}=\{\Gamma: \Gamma=\langle f, g\rangle \text { for some } \mathcal{R} \mathcal{P} \text {-pair }(f, g) \text { with } \\
& \left.\beta>-4, \beta^{\prime}>-4 \text {, and } \gamma<-\beta \beta^{\prime} / 4\right\} \text {. }
\end{aligned}
$$

The class $\mathcal{D}$ consists of the groups that have no invariant plane but can be generated by elements with real traces. Such groups are characterized in the following proposition:

Proposition 5.1 ([3, Proposition 1.1]). Let $\Gamma$ be a subgroup of $\operatorname{PSL}(2, \mathbb{C})$ generated by an $\mathcal{R} \mathcal{P}$-pair $(f, g)$. Then $\beta>-4, \beta^{\prime}>-4, \gamma<-\beta \beta^{\prime} / 4$, and $\gamma \neq 0$ if and only if $\Gamma$ satisfies all of the followings:

(1) $\Gamma$ is non-elementary.

(2) $\Gamma$ has no invariant plane (in particular, $\Gamma$ is not Fuchsian).

(3) Each of $f, g$ is either elliptic, parabolic, or hyperbolic.

Using this characterization, Klimenko and Kopteva classified the groups in $\mathcal{D}$ up to isomorphism (Theorem 2.1 of [3]), and listed the Kleinian orbifolds $Q(\Gamma)$ with $\Gamma \in \mathcal{D}$ (Theorem 3.1 of [3]). In section 3.3 of [3], they classified the $\Gamma$ in $\mathcal{D}$ for which $Q(\Gamma)$ is compact.

Definition 5.2 ([3, Section2]). We define the following group presentations:

(1) $G T[n, m ; q]=\left\langle f, g \mid f^{n}, g^{m},[f, g]^{q}\right\rangle$.

(2) $P H[n, m, q]=\left\langle x, y, z \mid x^{n}, y^{2}, z^{2},(x y)^{2},[x, y]^{m},(y x y z)^{q}\right\rangle$.

(3) $H[p ; n, m ; q]=\left\langle x, y, s \mid s^{2}, x^{n}, y^{m},\left(x y^{-1}\right)^{p},\left(s x s y^{-1}\right)^{q},\left(s x^{-1} y\right)^{2}\right\rangle$.

(4) $P[n, m, q]=\left\langle w, x, y, z \mid w^{n}, x^{2}, y^{2}, z^{2},(w x)^{2},(w y)^{2},(y z)^{2},(z x)^{q},(z w)^{m}\right\rangle$.

(5) $\operatorname{Tet}\left[p_{1}, p_{2}, p_{3} ; q_{1}, q_{2}, q_{3}\right]=\langle x, y, z| x^{p_{1}}, y^{p_{2}}, z^{p_{3}},\left(x y^{-1}\right)^{q_{3}},\left(y z^{-1}\right)^{q_{1}}$, $\left.\left(z x^{-1}\right)^{q_{2}}\right\rangle$. The group Tet $[2,2, n ; 2, q, m]$ is denoted by Tet $[n, m ; q]$ for simplicity.

(6) $\operatorname{GTet}_{1}[n, m, q]=\left\langle x, y, z \mid x^{n}, y^{2},(x y)^{m},[y, z]^{q},[x, z]\right\rangle$.

(7) GTet $2[n, m, q]=\left\langle x, y, z \mid x^{n}, y^{2},(x y)^{m},\left(x z^{-1} y^{-1} z y\right)^{q},[x, z]\right\rangle$.

(8) $\mathcal{S}_{2}[n, m, q]=\left\langle x, L \mid x^{n},\left(x L x L^{-1}\right)^{m},\left(x L^{2} x^{-1} L^{-2}\right)^{q}\right\rangle$. 
(9) $\mathcal{S}_{3}[n, m, q]=\left\langle x, L \mid x^{n},\left(x L x L^{-1}\right)^{m},\left(x L x L x L^{-2}\right)^{q}\right\rangle$.

(10) $R[n, m ; q]=\left\langle u, v \mid(u v)^{n},\left(u v^{-1}\right)^{m},[u, v]^{q}\right\rangle$.

Proposition 5.3 ([3, Section 3.3]). The compact orbifolds $Q=Q(\Gamma)=\mathbb{H}^{3} / \Gamma$ for $\Gamma \in \mathcal{D}$ are exactly those for which one of the following holds:

(1) $\pi_{1}^{\text {orb }}(Q) \cong P H[n, m, q]: n=4,3 \leq m \leq 5, q=3$.

(2) $\pi_{1}^{\text {orb }}(Q) \cong H[p ; n, m ; q]: \quad[p ; n, m, q]$ is $[2 ; 2,3 ; 5]$, or $[2 ; 2,5 ; 3]$, or $[2 ; 3,5 ; 2]$.

(3) $\pi_{1}^{\text {orb }}(Q) \cong T e t\left[2,3,3 ; 2,3, q_{3}\right]: q_{3}=4,5$; $\pi_{1}^{\text {orb }}(Q) \cong T e t[n, m ; q]: n=5, m=4,5, q=3$ or $n=m=3, q=5$

(4) $\pi_{1}^{\text {orb }}(Q) \cong P[n, m, q]$ :

$8 \leq n<\infty, n$ is even, $m=3, q=3,5$;

$4 \leq n<\infty, n$ is even, $m=5, q=3$.

(5) $\pi_{1}^{\text {orb }}(Q) \cong G$ Tet $_{2}[n, m, q]$ :

$n \geq 7$ is odd, $m=3,3 \leq q \leq 5$;

$n \geq 5$ is odd, $m=5, q=3$;

$n, m \geq 3$ are odd, $1 / n+1 / m<1 / 2, q=2$.

(6) $\pi_{1}^{\text {orb }}(Q) \cong \mathcal{S}_{3}[n, m, q]$ :

$n \geq 5$ is odd, $m=q=2$;

$n=5, m=2, q=3$;

$n=5, m=3, q=2$;

$n=3, m=4,5, q=2$.

(7) $\pi_{1}^{\text {orb }}(Q) \cong G \operatorname{Get}_{1}[n, m, q]: 7 \leq n<\infty, m=3, q=2$.

There are no compact orbifolds in the class $\mathcal{D}$ with $\pi_{1}^{\text {orb }}(Q) \cong G T[n, m ; q]$, $\mathcal{S}_{2}[n, m, q]$, or $R[n, 2 ; 2]$.

By Theorem 3.1 of [3], Figure 1 of [3] lists all $Q(\Gamma)$ for $\Gamma \in \mathcal{D}$. We need to know some notation to interpret the figures. Let $T(n)$ be a Seifert fibered solid torus obtained from a trivial solid torus $D^{2} \times \mathbb{S}^{1}$ by cutting it along $D^{2} \times\{x\}$ for some $x \in \mathbb{S}^{1}$, rotating one of the discs through $2 \pi / n$ and gluing back together. We denote a space obtained by gluing two copies of $T(n)$ along their boundaries fiber to fiber by $\mathcal{S}(n)$. See Section 3.2 of [3] for more details.

Figures 26, 27 and 28 list $Q(\Gamma)$, but they are a bit different from the original figures (Figure 1 of [3]). Klimenko and Kopteva used the concepts of "fat vertex" and "fat edge". Indeed "puncture", "boundary" and "cusp" can appear in Figure 1 of [3]. But in the cases of compact and closed orbifolds, no vertex can be a puncture and all edges are just singular loci with finite indices. So Figures 26, 27 and 28 are the same as the original figures when $\mathcal{O}$ is compact and closed (see Section 3.1 of [3] for details).

In the remaining part of this section, $M$ is a 3 -dimensional compact, closed $G$-manifold with genus two, where $G$ is a finite orientation preserving diffeomorphism, the Heegaard decomposition of $M$ is $\left(M: V_{1}, V_{2}\right)$, the corresponding handlebody orbifolds are $\mathcal{H}_{1}:=V_{1} / G$ and $\mathcal{H}_{2}:=V_{2} / G$ and $\mathcal{O}:=M / G$. 


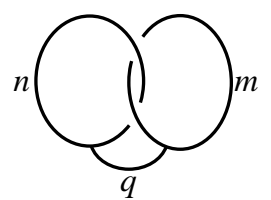

(a) $G T[n, m ; q]$

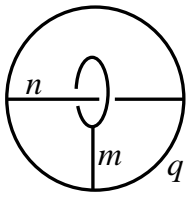

(b) $P \mathrm{H}[n, m, q]$

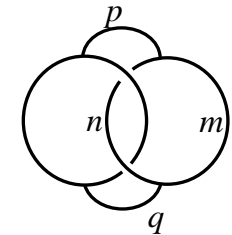

(c) $\mathrm{H}[p ; n, m ; q]$

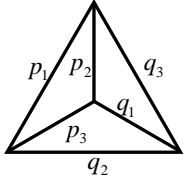

(d) $T \operatorname{et}\left[p_{1}, p_{2}, p_{3} ; q_{1}, q_{2}, q_{3}\right]$

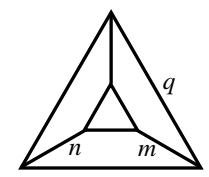

(e) $P[n, m, q]$

Figure 26. The underlying space is $\mathbb{S}^{3}$

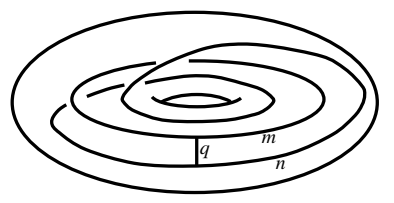

(f) Orbifolds embedded in $S(2)$; $\pi_{1}^{\mathrm{orb}}(\mathrm{Q}) \cong S_{2}[n, m, q]$

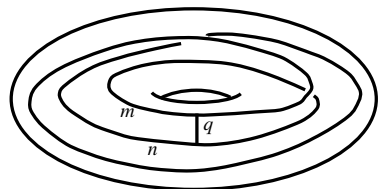

(h) Orbifolds embedded in $S$ (3); $\pi_{1}^{\mathrm{orb}}(\mathrm{Q}) \cong S_{3}[n, m, q]$

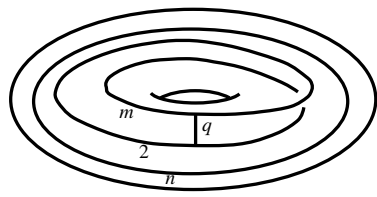

(g) Orbifolds embedded in $S$ (2); $\pi_{1}^{\mathrm{orb}}(\mathrm{Q}) \cong \operatorname{GTet}_{2}[n, m, q]$

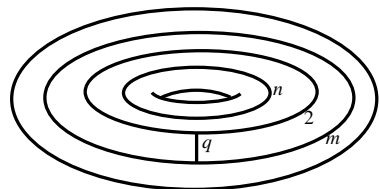

(i) Orbifolds embedded in $S^{2} \times S^{1}$; $\pi_{1}^{\mathrm{orb}}(\mathrm{Q}) \cong G T e t_{1}[n, m, q]$

Figure 27. The underlying space is $\mathcal{S}(n)$ or $\mathbb{S}^{2} \times \mathbb{S}^{1}$

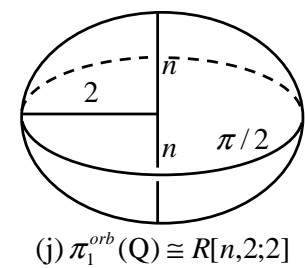

Figure 28. The underlying space is $\mathbb{R P}^{3}$

Figures 26, 27 and 28 are all from Figure 1 of [3]. 
If $\pi_{1}^{\text {orb }}(\mathcal{O}) \in \mathcal{D}$, then $\mathcal{O}$ is one of the orbifolds in Figures 26, 27, and 28 and also satisfies Theorem 3.3. In addition, if we use Proposition 5.3, then we may restrict the indices of the edges of the singular locus.

Lemma 5.4. Suppose that $\pi_{1}^{\text {orb }}(\mathcal{O}) \in \mathcal{D}$. Then the singular locus of $\mathcal{O}$ is a connected graph.

Proof. In Figures 26, 27, and 28 only connected singular loci appear when $\mathcal{O}$ is compact and closed.

By Lemma 5.4 we will consider only the cases of connected singular loci.

Theorem 5.5. If $\pi_{1}^{\text {orb }}(\mathcal{O}) \in \mathcal{D}$, then $G$ cannot be isomorphic to one of $\mathbb{Z}_{2}, \mathbb{Z}_{3}$, $\mathbb{Z}_{4}$, and $\mathbb{Z}_{6}$.

Proof. The singular loci of the listed cases are all knots or links, but there are no such singular loci in Figures 26, 27, and 28 in the cases of compact and closed $\mathcal{O}$.

Theorem 5.6. Suppose that $\pi_{1}^{\text {orb }}(\mathcal{O}) \in \mathcal{D}$. All the cases of $G \cong \mathbb{D}_{2}$ are impossible.

Proof. We will consider all the possible combinations of handlebody orbifolds.

Case 1. The corresponding $\mathbb{D}_{2}$-admissible graphs for $\mathcal{H}_{1}$ and $\mathcal{H}_{2}$ are both $\Gamma\left(\mathbb{Z}_{2}, 1, \mathbb{D}_{2}\right)$.

In this case, the underlying space is $\mathbb{S}^{3}$ by Lemma 4.2 and there are only two vertices of degree 3 . From Figure 26 the only possible case is $\pi_{1}^{\text {orb }}(\mathcal{O}) \cong$ $G T[n, m ; q]$, but it is impossible by Proposition 5.3.

Case 2. The corresponding $\mathbb{D}_{2}$-admissible graphs for $\mathcal{H}_{1}$ and $\mathcal{H}_{2}$ are both $\Gamma\left(\mathbb{D}_{2}, 2, \mathbb{D}_{2}, 2, \mathbb{D}_{2}\right)$.

The underlying space is $\mathbb{S}^{3}$ by Lemma 4.2 and there are exactly six vertices of degree 3 . From Figure 26 the possible case is $\pi_{1}^{\text {orb }}(\mathcal{O}) \cong P[n, m, q]$. But some edge must have index larger than 2 by Proposition 5.3, contradicting the fact that $\mathcal{O}$ is a union of $\mathcal{H}_{1}$ and $\mathcal{H}_{2}$ where the indices of edges of the singular locus are all 2.

Case 3. The corresponding $\mathbb{D}_{2}$-admissible graph of $\mathcal{H}_{1}$ is $\Gamma\left(\mathbb{Z}_{2}, 1, \mathbb{D}_{2}\right)$ and the corresponding $\mathbb{D}_{2}$-admissible graph of $\mathcal{H}_{2}$ is $\Gamma\left(\mathbb{D}_{2}, 2, \mathbb{D}_{2}, 2, \mathbb{D}_{2}\right)$.

The underlying space is $\mathbb{S}^{3}$ by Lemma 4.2 and the number of vertices of the singular locus is 4 . From Figure 26 the possible cases are $\pi_{1}^{\text {orb }}(\mathcal{O}) \cong$ $P H[n, m, q], \pi_{1}^{\text {orb }}(\mathcal{O}) \cong H[p ; n, m ; q]$ and $\pi_{1}^{\text {orb }}(\mathcal{O}) \cong T e t\left[p_{1}, p_{2}, p_{3} ; q_{1}, q_{2}, q_{3}\right]$. The indices of edges in the singular locus are all 2 , but this is impossible by Proposition 5.3.

Case 4. The corresponding $\mathbb{D}_{2}$-admissible graphs for $\mathcal{H}_{1}$ and $\mathcal{H}_{2}$ are all $\Gamma\left(\mathbb{D}_{2}, 2\right)$.

The singular locus consists of two disjoint loops and an edge which connects the base points of the loops. From Figures 26, 27 and 28 the possible cases are $\pi_{1}^{\text {orb }}(\mathcal{O}) \cong \mathcal{S}_{2}[n, m, q]$ and $\pi_{1}^{\text {orb }}(\mathcal{O}) \cong G T e t_{1}[n, m, q]$. Since $\mathcal{O}$ is a compact 
orbifold, $\pi_{1}^{\text {orb }}(\mathcal{O}) \cong \mathcal{S}_{2}[n, m, q]$ is impossible by Proposition 5.3. Since the indices of edges in the singular locus are all $2, \pi_{1}^{\text {orb }}(\mathcal{O}) \cong \operatorname{GTet}_{1}[n, m, q]$ is impossible by Proposition 5.3.

Theorem 5.7. Suppose that $\pi_{1}^{\text {orb }}(\mathcal{O}) \in \mathcal{D}$. All the cases of $G \cong \mathbb{D}_{3}$ are impossible.

Proof. We will consider all the possible combinations of handlebody orbifolds.

Case 1. The corresponding $\mathbb{D}_{3}$-admissible graphs for $\mathcal{H}_{1}$ and $\mathcal{H}_{2}$ are $\Gamma\left(\mathbb{Z}_{2}, 1\right.$, $\left.\mathbb{Z}_{3}\right)$.

The underlying space is $\mathbb{S}^{3}$ by Lemma 4.2 and the singular locus is a link, but there are no such singular loci in Figure 26 in the cases of compact and closed $\mathcal{O}$.

Case 2. The corresponding $\mathbb{D}_{3}$-admissible graphs for $\mathcal{H}_{1}$ and $\mathcal{H}_{2}$ are $\Gamma\left(\mathbb{D}_{3}, 2\right.$, $\left.\mathbb{D}_{3}\right)$.

The underlying space is $\mathbb{S}^{3}$ by Lemma 4.2 . The singular locus has exactly four vertices of degree 3 . Since no edge is separating edge, the possible cases are $\pi_{1}^{\text {orb }}(\mathcal{O}) \cong H[p ; n, m ; q]$ and $\pi_{1}^{\text {orb }}(\mathcal{O}) \cong T e t\left[p_{1}, p_{2}, p_{3} ; q_{1}, q_{2}, q_{3}\right]$ from Figure 26 . But all these cases are impossible since there must be an edge whose index is larger than 3 in each case by Proposition 5.3.

Case 3. The corresponding $\mathbb{D}_{3}$-admissible graph for $\mathcal{H}_{1}$ is $\Gamma\left(\mathbb{Z}_{2}, 1, \mathbb{Z}_{3}\right)$ and for $\mathcal{H}_{2}$ is $\Gamma\left(\mathbb{D}_{3}, 2, \mathbb{D}_{3}\right)$.

The underlying space is $\mathbb{S}^{3}$ by Lemma 4.2. The singular locus is connected and consists of two vertices of degree 3 and three edges which are all nonseparating. But no such singular loci are found in Figure 26.

Theorem 5.8. Suppose that $\pi_{1}^{\text {orb }}(\mathcal{O}) \in \mathcal{D}$. All the cases of $G \cong \mathbb{D}_{4}$ are impossible.

Proof. In this case, the corresponding $\mathbb{D}_{4}$-admissible graphs for $\mathcal{H}_{1}$ and $\mathcal{H}_{2}$ are $\Gamma\left(\mathbb{D}_{2}, 2, \mathbb{D}_{4}\right)$. The underlying space is $\mathbb{S}^{3}$ by Lemma 4.2 . There are exactly 4 vertices of degree 3 in the singular locus and one edge has index 4 and the other edges have index 2. Since no edge is separating edge, the possible cases are $\pi_{1}^{\text {orb }}(\mathcal{O}) \cong H[p ; n, m ; q]$ and $\pi_{1}^{\text {orb }}(\mathcal{O}) \cong T e t\left[p_{1}, p_{2}, p_{3} ; q_{1}, q_{2}, q_{3}\right]$ from Figure 26. But all these cases are impossible since there must be an edge of index 3 or 5 in each case by Proposition 5.3.

Theorem 5.9. Suppose that $\pi_{1}^{\text {orb }}(\mathcal{O}) \in \mathcal{D}$. All the cases of $G \cong \mathbb{D}_{6}$ are impossible.

Proof. In this case, the corresponding $\mathbb{D}_{6}$-admissible graphs for $\mathcal{H}_{1}$ and $\mathcal{H}_{2}$ are $\Gamma\left(\mathbb{D}_{2}, 2, \mathbb{D}_{3}\right)$. The underlying space is $\mathbb{S}^{3}$ by Lemma 4.2 . There are exactly 4 vertices of degree 3 in the singular locus and one edge has index 3 and the other edges have index 2 . Since no edge is separating, the possible cases are $\pi_{1}^{\text {orb }}(\mathcal{O}) \cong H[p ; n, m ; q]$ and $\pi_{1}^{\text {orb }}(\mathcal{O}) \cong \operatorname{Tet}\left[p_{1}, p_{2}, p_{3} ; q_{1}, q_{2}, q_{3}\right]$ from Figure 26 . But all these cases are impossible since there must be an edge whose index is larger than 3 in each case by Proposition 5.3. 
Corollary 5.10. Suppose that $M$ is compact, closed 3 -manifold and that $M$ is a $G$-manifold of genus 2 where $G$ is a finite orientation preserving diffeomorphism which acts on $M$. Then for the quotient orbifold $\mathcal{O}, \pi_{1}^{\text {orb }}(\mathcal{O}) \notin \mathcal{D}$.

Proof. By Theorems 5.5, 5.6, 5.7, 5.8 and 5.9, $\pi_{1}^{\text {orb }}(\mathcal{O}) \notin \mathcal{D}$ in all the possible cases from Theorem 3.3.

\section{References}

[1] M. Boileau and H. Zieschang, Nombre de ponts et générateurs méridiens des entrelacs de Montesinos, Comment. Math. Helv. 60 (1985) 270-279.

[2] W. D. Dunbar, Geometric orbifolds, Rev. Mat. Univ. Complut. Madrid 1 (1988), no. 1-3, $67-99$.

[3] E. Klimenko and N. Kopteva, Two-generator Kleinian orbifolds, arXiv:math/0606066.

[4] T. Kobayashi, Structures of the Haken manifolds with Heegaard splittings of genus two, Osaka J. Math. 21 (1984), no. 2, 437-455.

[5] D. McCullough, A. Miller, and B. Zimmermann, Group actions on handlebodies, Proc. London Math. Soc. (3) 59 (1989), no. 2, 373-416.

[6] W. H. Meeks and S.-T. Yau, The equivariant loop theorem for three-dimensional manifolds and a review of the existence theorems for minimal surfaces, The Smith conjecture (New York, 1979), 153-163, Pure Appl. Math., 112, Academic Press, Orlando, FL, 1984.

[7] A. Miller and B. Zimmermann, Large groups of symmetries of handlebodies, Proc. Amer. Math. Soc. 106 (1989), no. 3, 829-838.

[8] J. P. Serre, Trees, Translated from the French original by John Stillwell. Corrected 2nd printing of the 1980 English translation., Springer-Verlag, Berlin, 2003.

[9] B. Zimmermann, Genus actions of finite groups on 3-manifolds, Michigan Math. J. 43 (1996), no. 3, 593-610.

Department of Mathematics

Korea University

SeOul 136-701, Korea

E-mail address: pibonazi@gmail.com 